\title{
Fast and Precise Power Prediction for Combinational Circuits
}

Hongping Li, John K. Antonio, and Sudarshan K. Dhall

CS-TR-02-001

November 2002

Technical Report

\author{
School of Computer Science \\ University of Oklahoma \\ 200 Felgar Street \\ Norman OK 73019-6151
}

hongping@ou.edu, antonio@ou.edu, sdhall@ou.edu 


\title{
Fast and Precise Power Prediction for Combinational Circuits
}

\author{
Hongping Li, John K. Antonio, and Sudarshan K. Dhall \\ School of Computer Science, University of Oklahoma \\ 200 Felgar Street, Norman OK 73019-6151 \\ hongping@ou.edu, antonio@ou.edu,sdhall@ou.edu
}

\begin{abstract}
The power consumed by a combinational circuit is dictated by the switching activities of all signals associated with the circuit. An analytical approach is proposed for calculating signal activities for combinational circuits. The approach is based on a Markov chain signal model, and directly accounts for correlations present among the signals. The accuracy of the approach is verified by comparing signal activity values calculated using the proposed approach with corresponding values produced through simulation studies. It is also demonstrated that the proposed approach is accurate and computationally efficient.
\end{abstract}

Index Terms: Combinational Circuit, Probability, Activity, Markov Chain, Correlation Factor.

\section{Introduction}

Power consumption of integrated circuits (ICs) is of growing concern as more electronic devices are being deployed in mobile and portable applications, e.g., PDAs, mobile telephones, and other batterypowered electronic devices. As the functionality of such devices increases, so does the complexity and sophistication of the underlying circuits. More complexity and faster clock rates generally translate into higher power consumption for a given hardware implementation technology. Because battery 
technology has not improved at the same rate as IC technology, there is strong motivation to design circuits that are as power efficient as possible to extend battery life for portable devices.

Improvements in IC technologies (e.g., reduction in feature size) can reduce power requirements of a given circuit design. However, functionality and complexity of commercial devices generally increase from one generation to the next. So, the next generation device implemented with the next generation IC technology will generally have more functionality and complexity than the previous generation, and thus the issue of architectural design of the underlying circuits to be power efficient remains important.

Several similar and related approaches to this problem have been proposed in the past, including simulation-based [1] and analytical approaches [2, 3, 4]. A good survey of past approaches can be found in [5]. Generally, simulation-based approaches achieve high accuracy but require long execution times; in contrast, the analytical approaches are faster but are generally less accurate. In this paper a new analytical approach is proposed that achieves fast execution time and accuracy that is comparable with simulation-based methods. As explained below, the particular focus is on power consumption of circuits implemented in CMOS, but the proposed approach may be applicable for other technologies as well.

Power consumption in a CMOS circuit is primarily due to three types of current flow: leakage current, switching transient current, and load capacitance charging current [9]. The leakage current is associated with the imperfection of field effect transistors (FETs) that are used in CMOS devices. This type of current flow in CMOS technology is generally very small.

The switching transient current within CMOS gates is caused by a brief short circuit that can occur when the state of the complimentary gates change from on-to-off and off-to-on. This short circuit occurs when the complimentary MOSFETs are concurrently "on" for a brief transient period of time. 
The power loss due to switching transient current is dependent on the switching activity of the gate and is generally greater than leakage current.

The final type of current flow is load capacitance charging current. This is the current flow that is required to charge the capacitive load that is associated with a transistor gate, and occurs when the state of a gate changes. This is the dominant component of power consumption in CMOS devices, and is strongly dependent on signal switching activity.

Let $\Phi$ denote the set of all signals associated with a circuit. For each $s \in \Phi$, let $C(s)$ denote the capacitive load associated with signal $s$. Also, let $\alpha(s)$ denote the activity of signal $s$, which has a value between zero and one, and represents the signal's normalized average frequency relative to the frequency of a system clock, $f$. Thus, $f \alpha(s)$ gives the average frequency of signal $s$. Based on these assumptions and notation, the average power for a CMOS circuit operating at a voltage level of $V$ can be expressed as $[4,5]$ :

$$
\text { Power }_{\text {avg }}=\frac{1}{2} V^{2} f \sum_{s \in \Phi} C(s) \alpha(s) \text {. }
$$

The problem addressed in this report is to determine the activity of all signals of a combinational circuit given an appropriate probabilistic model for the primary input signals that drive the circuit. The signal model proposed in this report is based on a Markov chain. The signal activity is easily computed from the parameters associated with the proposed signal model. In the proposed approach, signals with known Markov chain representations are propagated through a model of the circuit to produce a Markov chain representation for the output of each gate in the circuit. Accuracy of the approach is verified by comparing signal activities produced by the proposed method with corresponding activities produced through simulation studies. When compared with other related approaches, a key aspect of 
the proposed approach is that correlations present among the signals due to re-convergent fan-out [6] are accounted for directly.

The rest of this report is organized as follows. In Section 2, an overview of related approaches is provided. The proposed approach, which utilizes a Markov chain signal model, is given in Section 3. The transformations and algorithm for applying this approach to a circuit is described and analyzed in Section 4. In Section 5, the accuracy of the proposed approach is verified through PSpice ${ }^{\circledR}$ circuit simulations, followed by conclusions in the final section.

\section{Previous Related Approaches}

\subsection{Basic Probabilistic Signal Parameter Definitions}

Signals in a combinational logic circuit can be treated in a probabilistic sense [1], i.e., for signal $x$, the probability that $x$ has logic value 1 is defined by $P(x)=P(x=1)$. Let $x(t), t \in(-\infty,+\infty)$, be a stochastic process that takes the values of logical 0 or logical 1 , transitioning from one to the other at random times. Generally, a stochastic process is said to be strict-sense stationary (SSS) if its statistical properties are invariable to a shift of time origin. Based on the assumptions of a SSS 0-1 mean-ergodic process $x(t)$, the following definitions are derived from [3].

Definition 2.1 (Signal Probability): The probability of a logic signal $x(t)$ is the average fraction of time that the signal is high and is given by

$$
P(x)=\lim _{T \rightarrow \infty} \frac{1}{T} \int_{\frac{-T}{2}}^{\frac{+T}{2}} x(t) d t
$$

Definition 2.2 (Signal Activity): Assume the average number of transitions in a time interval of length $T$ is given by $n(T)$. The signal activity of the corresponding signal $x(t)$ is given by 


$$
\alpha(x) \stackrel{\Delta}{=} \lim _{T \rightarrow \infty} \frac{n(T)}{T}
$$

\subsection{Signal Probability Calculation}

In [2], the concept of using probabilistic signal modeling for analysis of combinational circuits was first introduced. In this work, each signal is modeled with a single probabilistic parameter, $P(x)$, defining the probability of a signal having a logical value of one. The purpose is to calculate the probability parameter for all signals, given the probability parameters of the circuit's primary inputs. The motivation for this work originated from the area of pseudorandom testing, in which fault coverage and identification is achieved without resorting to exhaustive testing. Instead, by subjecting a circuit to a large number of randomly generated input signal vectors, one can deduce faults in the circuit by measuring the fraction of time that any given signal has logic value one. If any of the measured signal probabilities do not match calculated signal probabilities, then the possibility of a fault is present.

For signal $x$, the probability that $x$ has logic value 1 is defined by $P(x)=P(x=1)$. Two algorithms for calculating signal probabilities are introduced in [2]. These approaches require that a Boolean function expression associated with each signal be derived in terms of the primary inputs. Because the number of terms in these expressions can grow exponentially with the number of inputs, the complexity of these approaches can be prohibitive for practical circuits.

A computationally efficient algorithm for calculating signal probabilities is introduced in [7], named “Algorithm 1," which operates by propagating probability values through the gates of circuit, thereby drastically reducing the size of the Boolean functions that must be evaluated. Specifically, the probability of the output of a gate is expressed in terms of the probability values for the inputs to that 
gate (instead of the primary inputs of the entire circuit, as required by the approach in [2]). This algorithm is simple and fast - it has a linear complexity in the number of gates - but is not accurate for all classes of circuits.

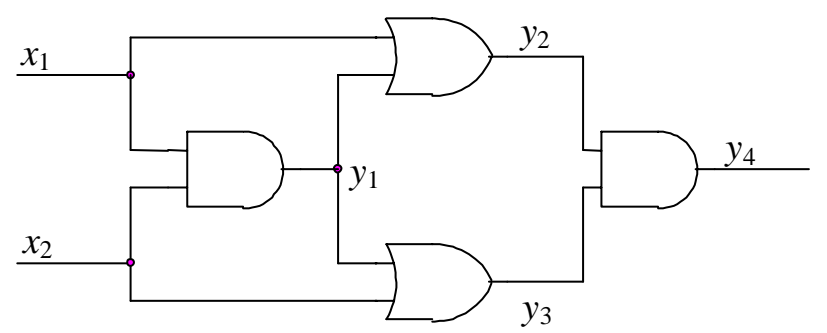

Figure 1. An example combinational circuit used to illustrate signal probability calculations (derived from [7]).

To illustrate the inaccuracies of Algorithm 1, assume in Figure 1 that the probabilities of primary inputs $x_{1}$ and $x_{2}$ are both 0.5. By applying Algorithm 1 of [7], the computed probabilities of the circuit's signals were calculated and are provided in Table 1.

Table 1. Comparison of actual signal probabilities and those calculated using Algorithm 1 in [7] for the circuit of Figure 1 with $P\left(x_{1}\right)=P\left(x_{2}\right)=1 / 2$.

\begin{tabular}{|l|c|c|c|c|}
\cline { 2 - 5 } \multicolumn{1}{c|}{} & $P\left(y_{1}\right)$ & $P\left(y_{2}\right)$ & $P\left(y_{3}\right)$ & $P\left(y_{4}\right)$ \\
\hline Actual & $1 / 4$ & $1 / 2$ & $1 / 2$ & $1 / 4$ \\
\hline Algorithm 1 in [7] & $1 / 4$ & $5 / 8$ & $5 / 8$ & $25 / 64$ \\
\hline
\end{tabular}

The problem with the accuracy of Algorithm 1 arises in circuits in which re-convergent fan-out signals are present. Re-convergent fan-out introduces functional dependencies and statistical correlations among the signals; however, Algorithm 1 assumes statistical independence among the inputs to each gate. For example, signals $y_{2}$ and $y_{3}$ in Figure 1 both depend on signal $x_{1}$ due to reconvergent fan-out. Thus, applying the algorithm to calculate $P\left(y_{4}\right)$ under the assumption that signals 
$y_{2}$ and $y_{3}$ are independent results in an error in the value calculated for $P\left(y_{4}\right)$, as shown in Table 1 . Similarly, the values calculated for $P\left(y_{2}\right)$ and $P\left(y_{3}\right)$ are also in error.

Another algorithm is proposed in [7] called the Weighted Averaging Algorithm (WAA), which generally achieves better accuracy than Algorithm 1 and has a comparable time complexity. However, the WAA still does not always produce correct values.

A method for accounting for signal probability correlations was developed in [6] named the correlation coefficient method (CCM). By using this approach, the probability of the output of a twoinput gate can be more accurately calculated, given the probabilities of the two inputs and an associated correlation factor associated with the two signals. In this algorithm, the correlation factor can also be calculated analytically by means of a set of basic propagation rules. By applying this CCM algorithm to the circuit shown in Figure 1, the values of $P\left(y_{1}\right), P\left(y_{2}\right), P\left(y_{3}\right)$, and $P\left(y_{4}\right)$ are properly calculated and correspond to the actual values shown in Table 1 . The time complexity of the CCM algorithm is $O\left(N^{2}\right)$ for a circuit with $N$ gates. ${ }^{1}$

\subsection{Signal Activity Calculation}

The above-described approaches of [2], [6], and [7] are concerned with determining the probabilities of signal values, not the probabilities of signal transitions, i.e., activities, which are necessary for estimating power consumption, refer to Eq. 1. An early approach for estimating signal activities was developed in [3], in which signals of a circuit are modeled to be mutually independent strict-sense-stationary (SSS) mean-ergodic 0-1 processes. Under these assumptions, the activity of a signal $y$ from a circuit with $n$-primary inputs can be expressed as

\footnotetext{
${ }^{1}$ Sharper time complexity results can be obtained; for example, it can be shown that a circuit with $\sqrt{N}$ levels has a complexity of $O\left(N^{3 / 2}\right)$
} 


$$
\alpha(y)=\sum_{i=1}^{n} P\left(\frac{\partial y}{\partial x_{i}}\right) \alpha\left(x_{i}\right)
$$

where $\frac{\partial y}{\partial x}$ is the Boolean difference of function $y$ with respect to $x_{i}$ and is defined by

$$
\begin{aligned}
\frac{\partial y}{\partial x_{i}}=\left.\left.y\right|_{x_{i}=1} \oplus y\right|_{x_{i}=0}= & y\left(x_{1}, \cdots, x_{i-1}, 1, x_{i+1}, \cdots, x_{n}\right) \\
& \oplus y\left(x_{1}, \cdots, x_{i-1}, 0, x_{i+1}, \cdots, x_{n}\right) .
\end{aligned}
$$

Intuitively, the Boolean difference $\frac{\partial y}{\partial x_{i}}$ defines whether a transition of signal $x_{i}$ will cause a transition in output signal $y$. Specifically, if the Boolean difference function evaluates to one, then a transition of signal $x_{i}$ causes a transition in $y$; if the Boolean difference function evaluates to zero, then a transition of signal $x_{i}$ does not cause a transition in $y$. So, the probability of the Boolean difference function, $P\left(\frac{\partial y}{\partial x_{i}}\right)$, defines the probability that a change in $y$ will occur given that there is a change in $x_{i}$. As an example of how to evaluate Eq. 2, consider a simple case of a three-input AND function in which $y=x_{1} x_{2} x_{3}$.

$$
\begin{gathered}
\alpha(y)=\sum_{i=1}^{3} P\left(\frac{\partial y}{\partial x_{i}}\right) \alpha\left(x_{i}\right), \\
\frac{\partial y}{\partial x_{1}}=\left.\left.y\right|_{x_{1}=1} \oplus y\right|_{x_{1}=0}=\left(1 \cdot x_{2} \cdot x_{3}\right) \oplus\left(0 \cdot x_{2} \cdot x_{3}\right)=x_{2} x_{3},
\end{gathered}
$$

and similarly

$$
\begin{aligned}
& \frac{\partial y}{\partial x_{2}}=x_{1} x_{3}, \\
& \frac{\partial y}{\partial x_{3}}=x_{1} x_{2} .
\end{aligned}
$$


Thus,

$$
\alpha(y)=P\left(x_{2} x_{3}\right) \alpha\left(x_{1}\right)+P\left(x_{1} x_{3}\right) \alpha\left(x_{2}\right)+P\left(x_{1} x_{2}\right) \alpha\left(x_{3}\right)
$$

Because $x_{1}, x_{2}$, and $x_{3}$ are mutually independent, we can further simplify the probability terms as follows:

$$
\begin{aligned}
\alpha(y) & =P\left(x_{2}\right) P\left(x_{3}\right) \alpha\left(x_{1}\right)+P\left(x_{1}\right) P\left(x_{3}\right) \alpha\left(x_{2}\right) \\
& +P\left(x_{1}\right) P\left(x_{2}\right) \alpha\left(x_{3}\right)
\end{aligned}
$$

The above expression is readily evaluated using the values of $P\left(x_{i}\right)$ and $\alpha\left(x_{i}\right)$, which are the known probabilities and activities of the primary input signals.

Although the calculation of the probability of the Boolean difference terms, i.e., $P\left(\frac{\partial y}{\partial x_{i}}\right)$, for the above example was relatively straightforward, this calculation can be complicated for large and complex circuits. In [3], the calculation of these terms is accomplished by first representing the nodes of the circuit with a binary decision diagram (BDD) $[3,5]$. In practice, the BDD approach often achieves linear or near linear time complexity; however, in the worst case the complexity can grow exponentially with the number of gates.

It is noted in [4] that Eq. 2, i.e., the approach described in [3], fails to consider the effect of simultaneous switching of gate inputs. Figure 2 shows an example of how simultaneous switching of inputs to a logic gate affects the activity of the output node. As shown in the figure, if the two input signals always switch simultaneously, then the output signal of the XOR gate will have an activity of zero, even though the probability and activity terms in Eq. 2 are nonzero [4]. This example is an extreme case, but is given to illustrate the importance of considering simultaneous switching. 


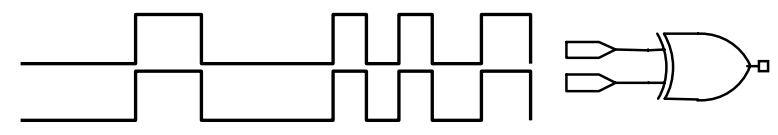

Figure 2. Example to illustrate the effect of simultaneous switching (derived from [4]).

Each Boolean difference term associated with Eq. 2 describes an input-switching event in which exactly one of the inputs makes a transition. Thus, Eq. 2 does not account for events involving simultaneous switching of two or more of the input signals. The concept of the generalized Boolean difference was introduced in [4] to account for simultaneous switching, and is denoted as follows:

$$
\begin{aligned}
\frac{\partial y^{k} \mid b_{i_{1}}, b_{i_{2}}, \ldots, b_{i_{k}}}{\partial x_{i_{1}} \partial x_{i_{2}} \ldots \partial x_{i_{k}}}= & \left(y \mid x_{i_{1}}=b_{i_{1}}, x_{i_{2}}=b_{i_{2}}, \ldots x_{i_{k}}=b_{i_{k}}\right) \\
& \oplus\left(y \mid x_{i_{1}}=\overline{b_{i_{1}}}, x_{i_{2}}=\overline{b_{i_{2}}}, \ldots, x_{i_{k}}=\overline{b_{i_{k}}}\right),
\end{aligned}
$$

where $k$ is a positive integer, $x_{i_{j}}, j=1,2, \ldots, k$, are distinct mutually independent primary inputs of $y$, and $b_{i_{j}}$ are binary values of 0 or 1 . Note that if the generalized Boolean difference evaluates to one, then the simultaneous transitions of signals $\left(x_{i_{1}}, x_{i_{2}}, \ldots, x_{i_{k}}\right)$ from $\left(b_{i_{1}}, b_{i_{2}}, \ldots, b_{i_{k}}\right)$ to $\left(\overline{b_{i_{1}}}, \overline{b_{i_{2}}}, \ldots, \overline{b_{i_{k}}}\right)$ or from $\left(\overline{b_{i_{1}}}, \overline{b_{i_{2}}}, \ldots, \overline{b_{i_{k}}}\right)$ to $\left(b_{i_{1}}, b_{i_{2}}, \ldots, b_{i_{k}}\right)$ will cause a transition at $y$.

Eq. 2 is adapted in [4] using the generalized Boolean difference concept to account for simultaneous switching, resulting in:

$$
\begin{aligned}
\alpha(y) & =\sum_{i=1}^{n} P c\left(\frac{\partial y}{\partial x_{i}}\right)\left(\alpha\left(x_{i}\right) \prod_{\substack{j \neq i \\
1 \leq j \leq n}}\left[1-\alpha\left(x_{i}\right)\right]\right) \\
& +\frac{1}{2}\left\{\sum\left[P c\left(\frac{\left.\partial^{2} y\right|_{00}}{\partial x_{i} \partial x_{j}}\right)+P c\left(\frac{\left.\partial^{2} y\right|_{01}}{\partial x_{i} \partial x_{j}}\right)\right]\left(\alpha\left(x_{i}\right) \alpha\left(x_{j}\right) \prod_{l \in\{1,2, \ldots, n\}-\{i, j\}}\left[1-\alpha\left(x_{l}\right)\right]\right)\right\}+\ldots \\
& +\frac{1}{2^{n-1}}\left[P c\left(\frac{\left.\partial^{n} y\right|_{00 \ldots .}}{\partial x_{1} \partial x_{2} \ldots \partial x_{n}}\right)+P c\left(\frac{\left.\partial^{n} y\right|_{00 \ldots 1}}{\partial x_{1} \partial x_{2} \ldots \partial x_{n}}\right)+\ldots+P c\left(\frac{\left.\partial^{n} y\right|_{01 \ldots 1}}{\partial x_{1} \partial x_{2} \ldots \partial x_{n}}\right)\right]\left(\prod_{l=1}^{n} \alpha\left(x_{l}\right)\right) .
\end{aligned}
$$


where $P c\left(\frac{\partial y}{\partial x_{i}}\right), P c\left(\frac{\left.\partial^{2} y\right|_{00}}{\partial x_{i} \partial x_{j}}\right), \ldots, P c\left(\frac{\left.\partial^{n} y\right|_{01 \ldots 1}}{\partial x_{1} \partial x_{2} \cdots \partial x_{n}}\right)$ are conditional probabilities of the generalized

Boolean differences under the condition that only the indicated inputs simultaneously switch, and the rest do not. Details on how to calculate these conditional probabilities can be found in [4].

Applying Eq. 10 to the same three-input AND function $y=x_{1} x_{2} x_{3}$ used earlier results in the following:

$$
\begin{aligned}
\alpha(y)= & P\left(x_{1}\right) P\left(x_{2}\right) \alpha\left(x_{3}\right)+P\left(x_{1}\right) P\left(x_{3}\right) \alpha\left(x_{2}\right) \\
& +P\left(x_{2}\right) P\left(x_{3}\right) \alpha\left(x_{1}\right) \\
& -\frac{1}{2} P\left(x_{1}\right) \alpha\left(x_{2}\right) \alpha\left(x_{3}\right)-\frac{1}{2} P\left(x_{2}\right) \alpha\left(x_{1}\right) \alpha\left(x_{3}\right) \\
& -\frac{1}{2} P\left(x_{3}\right) \alpha\left(x_{1}\right) \alpha\left(x_{2}\right)+\frac{1}{4} \alpha\left(x_{1}\right) \alpha\left(x_{2}\right) \alpha\left(x_{3}\right)
\end{aligned}
$$

Observe that all of the terms of Eq. 8 also appear as terms in Eq. 11. The rest of the terms in Eq. 11 arise due to the generalized Boolean difference factors that account for simultaneous switching. In general, the approach of Eq. 10 yields more accurate results than Eq. 2. However, the overall complexity associated with evaluating Eq. 10 is generally much larger than that of Eq. 2. This high complexity is due to a potentially large number of terms (exponential in the number of inputs) and the complexity associated with evaluating the conditional probabilities. For more discussion about the complexity and techniques for calculating the conditional probabilities, refer to [4].

\subsection{Summary of Previous Related Approaches}

The signal model for the approaches overviewed in Subsection 2.2 is based on a single probability parameter $[2,6,7]$. Although this probability parameter is not directly used in calculating a circuit's power consumption, refer to Eq. 1, it is a necessary component for the signal model common to the 
approaches of Subsection 2.3, which utilize both signal probability and signal activity parameters [3, 4].

The approaches of [2], [3], and [4] can have high computational complexities because the number of terms in the underlying equations/transformations can grow exponentially with the number of primary inputs to the circuit. In [7], a trade-off between computational complexity and resulting accuracy is illustrated in the context of the underlying equations/transformations introduced in [2]. In particular, an approximate approach is defined in [7] in which the transformations of [2] are applied in a "gate-bygate" fashion. Thus, instead of deriving the transformation for a signal's probability parameter in terms of the circuit's primary inputs, it is derived in terms of the immediate inputs to the logic gate associated with the signal. This approach greatly reduces the computational complexity, but introduces error in the calculated probability parameters for circuits with re-convergent fan-out.

Similar trade-offs between computational complexity and accuracy are possible relative to the evaluation of Eq. 2 and Eq. 10 (associated with [3] and [4], respectively). Instead of deriving a signal's logic function in terms of the circuit's primary inputs, the parameters to the immediate inputs the signal's logic gate can be used. Again, this type of "gate-by-gate" technique will generally introduce error because it does not account for correlations present among the internal signals that drive the gates within the circuit.

The approach of [6] is a fast and accurate "gate-by-gate" technique for calculating a signal's probability parameter. It introduces the concept of a correlation factor to account for and appropriately adjust the transformation for correlated inputs to a gate.

\section{Markov Chain Signal Model}




\subsection{Preliminaries}

In this section we introduce a signal model that is based on a Markov chain having three event parameters. It is shown that the proposed Markov chain model is equivalent to the two-parameter probability/activity signal model of [3] and [4]. The advantage of modeling signals with Markov chains is that it makes it possible to compute correlations between signals related to both probability and activity.

The approach derived here can be viewed as a generalization of the approach in [6]. Instead of tracking a correlation factor for the single probability parameter model, transformations for correlation factors associated with the three parameters of the Markov model are derived. This ultimately leads to a fast and accurate "gate-by-gate" algorithm for calculating signal probabilities and activities.

As illustrated in Figure 3, the proposed Markov chain signal model has three event parameters for signal $A$. The event denoted by $A$ represents the signal being in state 1 , and $A_{1}$ and $A_{2}$ represent the events that there is a transition from state 0 to 1 and from state 1 to 0 , respectively. Note that the probability of event $A$ is denoted by $P(A)$, and is equivalent to the signal probability defined in the previous section.

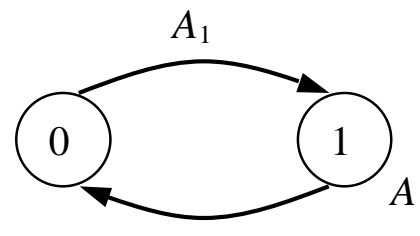

$A_{2}$

Figure 3. Proposed Markov chain signal model.

For notational convenience and clarity, we will denote the value of $P(A)$ as $p_{A}$ (for the value of the probability of signal $A$ ) and the value of the activity $\alpha(A)$ as $\alpha_{A}$ (for the value of the activity of signal 
A) throughout the rest of the paper. Using these notations and applying basic properties of Markov chains along with the definition of signal activity, the following expressions can be derived for $P(A)$, $P\left(A_{1}\right)$ and $P\left(A_{2}\right)$ :

$$
\begin{gathered}
P(A)=p_{A}, \\
P\left(A_{1}\right)=\frac{\alpha_{A}}{2\left(1-p_{A}\right)}, \\
P\left(A_{2}\right)=\frac{\alpha_{A}}{2 p_{A}} .
\end{gathered}
$$

Thus, if the values of both the probability and activity parameters of a signal are known (i.e., $p_{A}$ and $\left.\alpha_{A}\right)$, then the probabilities of the three events associated with the proposed Markov model for the signal are completely determined. Likewise, knowing the probability values of the three parameters of the Markov model fully determines the probability and activity parameters of the signal.

In order to define correlations between two signals modeled with Markov chains, some basic definitions are needed. Let $A$ and $B$ denote two events and let $P(A B)$ denote the probability of both $A$ and $B$ occurring. From basic probability theory [8], $P(A B)=P(A / B) P(B)$, where $P(A / B)$ represents the probability of $A$ given $B$. Also, the correlation coefficient of two events $A$ and $B$ is defined as

$$
\rho_{A B}=\frac{\sigma_{A B}}{\sigma_{A} \sigma_{B}},
$$

where $\sigma_{A B}$ is the covariance and $\sigma_{A}$ and $\sigma_{B}$ are the positive square roots of the variances of $A$ and $B$. It can be shown that

$$
\rho_{A B}=\frac{P(A B)-P(A) P(B)}{\sqrt{P(A)(1-P(A))} \sqrt{P(B)(1-P(B))}} .
$$

In order to simplify later derivations, it is convenient to define the correlation factor $C_{A B}$ of two events $A$ and $B$ as 


$$
C_{A B}=\frac{P(A B)}{P(A) P(B)}=\frac{P(A / B)}{P(A)}=\frac{P(B / A)}{P(B)} .
$$

By applying Eq. 17 to Eq. 16, the following relationship can be derived:

$$
\rho_{A B}=\frac{P(A)}{\sqrt{P(A)(1-P(A))}} \frac{P(B)}{\sqrt{P(B)(1-P(B))}}\left(C_{A B}-1\right) .
$$

Thus, $C_{A B}$ is related to $\rho_{A B}$ through scaling and shifting. The value of $\rho_{A B}$, by definition [8], is a real number in the interval $[-1,1]$; therefore, according to Eq. $18, C_{A B}$ takes on real non-negative values. Also, $\rho_{A B}=0$ corresponds to $C_{A B}=1$, and indicates that the events $A$ and $B$ are mutually independent. Similarly, $\rho_{A B}<0$ (i.e., $A$ and $B$ are negatively correlated) corresponds to $0 \leq C_{A B}<1$, and $\rho_{A B}>0$ (i.e., $A$ and $B$ are positively correlated) corresponds to $C_{A B}>1$.

\subsection{Markov Chain Model for Basic Logic Gates}

The focus in this subsection is on deriving the Markov chain model for the output of a basic logic gate in which the Markov chain models of the input signals are known. The simple case of a NOT gate is considered first followed by the analysis of two-input basic logic gates.

For a NOT gate with input $A$, the Boolean output function is given by $Y=\bar{A}$. From Figure 3 , it is clear that the Markov model for $Y$ is given by

$$
P(Y)=1-P(A), \quad P\left(Y_{1}\right)=P\left(A_{2}\right), P\left(Y_{2}\right)=P\left(A_{1}\right) .
$$

Consider now the case of a two-input basic logic gate, as shown in Figure 4. Assuming the Markov chain models of $A$ and $B$ are known, the objective is to derive the Markov chain model for output signal $Y$. 


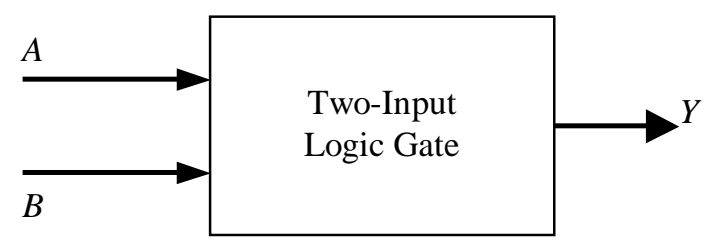

Figure 4. Generic two-input logic gate.

A key to deriving the Markov chain model for signal $Y$ of Figure 4 is to represent the state transition diagram associated with the gate's two inputs, as shown in Figure 5. The four states in the figure correspond to the four input combinations for the two inputs. The first digit of each state label corresponds to the value of $A$, and the second to the value of $B$, e.g., the state labeled " 01 " corresponds to $A=0$ and $B=1$. Although not labeled on the figure, the directed edges represent transition events. To illustrate the notation to label transition events, " $00 \rightarrow 10$ " will be used to represent the event that input signal $A$ transitions from 0 to 1 and signal $B$ stays in state 0 .

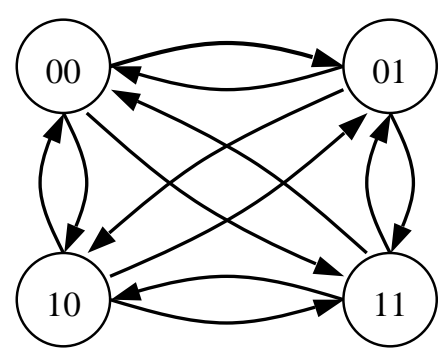

Figure 5. State transition diagram for inputs $A$ and $B$ of Figure 4.

The known parameters of the Markov chain models for signals $A$ and $B$ are given by $P(A), P\left(A_{1}\right)$, $P\left(A_{2}\right), P(B), P\left(B_{1}\right)$, and $P\left(B_{2}\right)$. Also assumed to be known are the correlation factors for pairs of 
events associated with the Markov chain models for the inputs. ${ }^{2}$ From Eq. 17 note that $P(A B)=$ $P(A) P(B) C_{A B}$, where $C_{A B}$ is the correlation factor associated with events $A$ and $B$. Similarly, the correlation factor $C_{A_{1} B_{2}}$ enables the calculation of $P\left(A_{1} B_{2}\right)$ using the fact that $P\left(A_{1} B_{2}\right)=P\left(A_{1}\right) P\left(B_{2}\right) C_{A_{1} B_{2}}$. Recall from Eq. 18 that independent events correspond to a correlation factor of unity.

Given the Markov chain models for signals $A$ and $B$ (and the corresponding correlation factors) it is possible to derive the probability associated with every event shown in the state transition diagram of Figure 5. To illustrate, consider the probability of event $00 \rightarrow 01$ :

$$
\begin{aligned}
P(00 & \rightarrow 01)=P\left(\overline{A_{1}} B_{1}\right) \\
& =P\left(\overline{A_{1}} / B_{1}\right) P\left(B_{1}\right) \\
& =\left[1-P\left(A_{1} / B_{1}\right)\right] P\left(B_{1}\right) \\
& =P\left(B_{1}\right)-P\left(A_{1} / B_{1}\right) P\left(B_{1}\right) \\
& =P\left(B_{1}\right)-P\left(A_{1} B_{1}\right) \\
& =P\left(B_{1}\right)-P\left(A_{1}\right) P\left(B_{1}\right) C_{A_{1} B_{2}}
\end{aligned}
$$

Expressions for the probabilities of all events associated with the state transition diagram of Figure 5 can be derived similarly; a complete tabulation of these expressions are given in Table 2 .

Table 2. Probabilities of events associated with Figure 5.

\begin{tabular}{|c|l|}
\hline Event & \multicolumn{1}{|c|}{ Probability } \\
\hline state 00 & $P(00)=1-p_{A}-p_{B}+p_{A} p_{B} C_{A B}$ \\
\hline state 01 & $P(01)=p_{B}-p_{A} p_{B} C_{A B}$ \\
\hline state 10 & $P(10)=p_{A}-p_{A} p_{B} C_{A B}$ \\
\hline state 11 & $P(11)=p_{A} p_{B} C_{A B}$ \\
\hline $00 \rightarrow 00$ & $P(00 \rightarrow 00)=1-\frac{\alpha_{A}}{2\left(1-p_{A}\right)}-\frac{\alpha_{B}}{2\left(1-p_{B}\right)}+\frac{\alpha_{A}}{2\left(1-p_{A}\right)} \frac{\alpha_{B}}{2\left(1-p_{B}\right)} C_{A_{B} B_{1}}$ \\
\hline $00 \rightarrow 01$ & $P(00 \rightarrow 01)=\frac{\alpha_{B}}{2\left(1-p_{B}\right)}\left(1-\frac{\alpha_{A}}{2\left(1-p_{A}\right)} C_{A_{1} B_{1}}\right)$ \\
\hline
\end{tabular}

${ }^{2}$ Deriving transformations to determine correlations factors associated with pairs of signals will be discussed in Subsection 3.3; for purposes of the present subsection they are assumed to be known. 


\begin{tabular}{|l|l|}
\hline $00 \rightarrow 11$ & $P(00 \rightarrow 11)=\frac{\alpha_{A}}{2\left(1-p_{A}\right)} \frac{\alpha_{B}}{2\left(1-p_{B}\right)} C_{A_{1} B_{1}}$ \\
\hline $00 \rightarrow 10$ & $P(00 \rightarrow 10)=\frac{\alpha_{A}}{2\left(1-p_{A}\right)}\left(1-\frac{\alpha_{B}}{2\left(1-p_{B}\right)} C_{A_{1} B_{1}}\right)$ \\
\hline $01 \rightarrow 00$ & $P(01 \rightarrow 00)=\frac{\alpha_{B}}{2 p_{B}}\left(1-\frac{\alpha_{A}}{2\left(1-p_{A}\right)} C_{A_{1} B_{2}}\right)$ \\
\hline $01 \rightarrow 01$ & $P(01 \rightarrow 01)=1-\frac{\alpha_{B}}{2 p_{B}}-\frac{\alpha_{A}}{2\left(1-p_{A}\right)}\left(1-\frac{\alpha_{B}}{2 p_{B}} C_{A_{1} B_{2}}\right)$ \\
\hline $01 \rightarrow 10$ & $P(01 \rightarrow 10)=\frac{\alpha_{A}}{2\left(1-p_{A}\right)} \frac{\alpha_{B}}{2 p_{B}} C_{A_{1} B_{2}}$ \\
\hline $01 \rightarrow 11$ & $P(01 \rightarrow 11)=\frac{\alpha_{A}}{2\left(1-p_{A}\right)}\left(1-\frac{\alpha_{B}}{2 p_{B}} C_{A_{1} B_{2}}\right)$ \\
\hline $10 \rightarrow 00$ & $P(10 \rightarrow 00)=\frac{\alpha_{A}}{2 p_{A}}\left(1-\frac{\alpha_{B}}{2\left(1-p_{B}\right)} C_{A_{2} B_{1}}\right)$ \\
\hline $10 \rightarrow 01$ & $P(10 \rightarrow 01)=\frac{\alpha_{A}}{2 p_{A}} \frac{\alpha_{B}}{2\left(1-p_{B}\right)} C_{A_{2} B_{1}}$ \\
\hline $10 \rightarrow 10$ & $P(10 \rightarrow 10)=1-\frac{\alpha_{A}}{2 p_{A}}-\frac{\alpha_{B}}{2\left(1-p_{B}\right)}+\frac{\alpha_{A}}{2 p_{A}} \frac{\alpha_{B}}{2\left(1-p_{B}\right)} C_{A_{2} B_{1}}$ \\
\hline $10 \rightarrow 11$ & $P(10 \rightarrow 11)=\frac{\alpha_{B}}{2\left(1-p_{B}\right)}\left(1-\frac{\alpha_{A}}{2 p_{A}} C_{A_{2} B_{1}}\right)$ \\
\hline $11 \rightarrow 00$ & $P(11 \rightarrow 00)=\frac{\alpha_{A}}{2 p_{A}} \frac{\alpha_{B}}{2 p_{B}} C_{A_{2} B_{2}}$ \\
\hline $11 \rightarrow 01$ & $P(11 \rightarrow 01)=\frac{\alpha_{A}}{2 p_{A}}\left(1-\frac{\alpha_{B}}{2 p_{B}} C_{A_{2} B_{2}}\right)$ \\
\hline $11 \rightarrow 10$ & $P(11 \rightarrow 10)=\frac{\alpha_{B}}{2 p_{B}}\left(1-\frac{\alpha_{A}}{2 p_{A}} C_{A_{2} B_{2}}\right)$ \\
\hline $11 \rightarrow 11$ & $P(11 \rightarrow 11)=1-\frac{\alpha_{A}}{2 p_{A}}-\frac{\alpha_{B}}{2 p_{B}}+\frac{\alpha_{A}}{2 p_{A}} \frac{\alpha_{B}}{2 p_{B}} C_{A_{2} B_{2}}$ \\
\hline
\end{tabular}

Deriving a Markov chain model for $Y$ of Figure 4 depends on the particular function of the gate. To illustrate how to determine the Markov chain model for $Y$, consider the specific example of an AND gate, i.e., $Y=A B$. For an AND gate, the output takes on logic value 1 if and only if both inputs are 1 . Thus, 


$$
P(Y)=P(11)=p_{A} p_{B} C_{A B} .
$$

The event $Y_{1}$ is associated with three events from Figure 5, namely: $00 \rightarrow 11,01 \rightarrow 11$, and $10 \rightarrow 11$. Thus, equality can be established as follows:

$$
\begin{gathered}
P(\bar{Y}) P\left(Y_{1}\right)=P(00) P(00 \rightarrow 11)+P(01) P(00 \rightarrow 11) \\
+P(01) P(00 \rightarrow 11)
\end{gathered}
$$

Solving Eq. 22 for $P\left(Y_{1}\right)$ and using Eqs. 12 through 14 results in the following expression:

$$
\begin{aligned}
P\left(Y_{1}\right)= & \left(\frac{1}{2} \lambda_{A} p_{B} \alpha_{A}+\frac{1}{2} \lambda_{B} p_{A} \alpha_{B}\right) /\left(1-p_{A} p_{B} C_{A B}\right) \\
& -\left[\frac{1}{4}\left(\lambda_{A} C_{A_{1} B_{2}}+\lambda_{B} C_{A_{2} B_{1}}-\lambda C_{A_{1} B_{1}}\right) \alpha_{A} \alpha_{B} /\left(1-p_{A} p_{B} C_{A B}\right)\right]
\end{aligned}
$$

where $\lambda_{A}=\frac{1-p_{A} C_{A B}}{1-p_{A}}, \lambda_{B}=\frac{1-p_{B} C_{A B}}{1-p_{B}}$ and $\lambda=\frac{1-p_{A}-p_{B}+p_{A} p_{B} C_{A B}}{\left(1-p_{A}\right)\left(1-p_{B}\right)}$.

Derivation for $P\left(Y_{2}\right)$ follows in a similar fashion and can be expressed as

$$
P\left(Y_{2}\right)=\frac{\alpha_{1}}{2 p_{1}}+\frac{\alpha_{2}}{2 p_{2}}-\frac{\alpha_{1}}{2 p_{1}} \frac{\alpha_{2}}{2 p_{2}} C_{A_{2} B_{2}} .
$$

Derivations of $P(Y), P\left(Y_{1}\right)$, and $P\left(Y_{2}\right)$ for two-input $\mathrm{OR}$ and $\mathrm{XOR}$ gates, i.e. $Y=A+B$ and $Y=A \oplus B$ respectively, are similar to the above derivation for the AND gate and the results are shown in Table 3. To reduce the notational burden, the formulas in Table 3 are expressed in terms of signal probabilities and activities instead of the Markov chain parameters (i.e., Eqs. 12 to 14 were applied).

Table 3. Formulas for computing Markov chain parameters for the output of basic gates.

\begin{tabular}{|c|c|c|c|}
\hline Gate & $P(Y)$ & $P\left(Y_{1}\right)$ & $P\left(Y_{2}\right)$ \\
\hline NOT & $1-p_{A}$ & $P\left(A_{2}\right)$ & $P\left(A_{1}\right)$ \\
$Y=\bar{A}$ & & \\
\hline
\end{tabular}




\begin{tabular}{|c|c|c|c|}
\hline $\begin{array}{l}\text { AND } \\
Y=A B\end{array}$ & $p_{A} p_{B} C_{A B}$ & $\begin{array}{l}\left(\frac{1}{2} \lambda_{A} p_{B} \alpha_{A}+\frac{1}{2} \lambda_{B} p_{A} \alpha_{B}\right) /\left(1-p_{A} p_{B} C_{A B}\right) \\
-\left[\frac{1}{4}\left(\lambda_{A} C_{A_{1} B_{2}}+\lambda_{B} C_{A_{2} B_{1}}-\lambda C_{A_{1} B_{1}}\right) \alpha_{A} \alpha_{B} /\left(1-p_{A} p_{B} C_{A B}\right)\right]\end{array}$ & $\frac{\alpha_{A}}{2 p_{A}}+\frac{\alpha_{B}}{2 p_{B}}-\frac{\alpha_{A}}{2 p_{A}} \frac{\alpha_{B}}{2 p_{B}} C_{A_{2} B_{2}}$ \\
\hline $\begin{array}{l}\mathrm{OR} \\
Y=A+B\end{array}$ & $\begin{array}{l}p_{A}+p_{B} \\
-p_{A} p_{B} C_{A B}\end{array}$ & $\begin{array}{l}\frac{\alpha_{A}}{2\left(1-p_{A}\right)}+\frac{\alpha_{B}}{2\left(1-p_{B}\right)} \\
-\frac{\alpha_{A}}{2\left(1-p_{A}\right)} \frac{\alpha_{B}}{2\left(1-p_{B}\right)} C_{A_{1} B_{1}}\end{array}$ & \begin{tabular}{|c}
$\frac{\left(1-p_{A}-p_{B}+p_{A} p_{B} C_{A B}\right)}{4\left(1-p_{A}\right)\left(1-p_{B}\right)\left(p_{A}+p_{B}-p_{A} p_{B} C_{A B}\right)}$ \\
$\times \frac{\alpha_{A}\left(1-p_{B}\right)+\left(1-p_{A}\right) \alpha_{B}-\alpha_{A} \alpha_{B} C_{A_{1} B_{1}}}{2\left(1-p_{A}\right)\left(1-p_{B}\right)\left(p_{A}+p_{B}-p_{A} p_{B} C_{A B}\right)}$
\end{tabular} \\
\hline $\begin{array}{l}\mathrm{XOR} \\
Y=A \oplus B\end{array}$ & $\begin{array}{l}p_{A}+p_{B} \\
-2 p_{A} p_{B} C_{A B}\end{array}$ & $\begin{array}{l}\frac{1}{2} \frac{\lambda-p_{B} \lambda+p_{B} C_{A B}}{1-p_{A}-p_{B}+2 p_{A} p_{B} C_{A B}} \alpha_{A} \\
+\frac{1}{2} \frac{\lambda-p_{A} \lambda+p_{A} C_{A B}}{1-p_{A}-p_{B}+2 p_{A} p_{B} C} \alpha_{B} \\
-\frac{1}{2} \frac{\lambda C_{A_{1} B_{1}}+C_{A_{2} B_{2}}}{1-p_{A}-p_{B}+2 p_{A} p_{B} C_{A B}} \alpha_{A} \alpha_{B}\end{array}$ & $\begin{array}{l}\frac{\frac{1}{2} p_{B} \lambda_{A}+\frac{1}{2}\left(1-p_{B}\right) \lambda_{B}}{p_{A}+p_{B}-2 p_{A} p_{B} C_{A B}} \alpha_{A} \\
+\frac{\frac{1}{2} p_{A} \lambda_{B}+\frac{1}{2}\left(1-p_{A}\right) \lambda_{A}}{p_{A}+p_{B}-2 p_{A} p_{B} C_{A B}} \alpha_{B} \\
-\frac{\frac{1}{2}\left(\lambda_{A} C_{A_{1} B_{2}}+\lambda_{B} C_{A_{2} B_{1}}\right)}{p_{A}+p_{B}-2 p_{A} p_{B} C_{A B}} \alpha_{A} \alpha_{B}\end{array}$ \\
\hline \multicolumn{4}{|c|}{$\lambda_{A}=\frac{1-p_{A} C_{A B}}{1-p_{A}} \quad \lambda_{B}=\frac{1-p_{B} C_{A B}}{1-p_{B}} \quad \lambda=\frac{1-p_{A}-p_{B}+p_{A} p_{B} C_{A B}}{\left(1-p_{A}\right)\left(1-p_{B}\right)}$} \\
\hline
\end{tabular}

Applying Eqs. 12 to 14, and using the parameter results listed in Table 3, the probability and activity values of the output signal $Y$ of these two-input AND, OR and XOR gates and the NOT gate can be derived and the results are shown in Table 4.

Table 4. Probability and activity values of output signals of basic gates.

\begin{tabular}{|c|c|c|}
\hline Gate & $p_{Y}$ & $\alpha_{Y}$ \\
\hline $\begin{array}{c}\text { NOT } \\
Y=\bar{A}\end{array}$ & $1-p_{A}$ & $\alpha_{A}$ \\
\hline $\begin{array}{c}\text { AND } \\
Y=A B\end{array}$ & $p_{A} p_{B} C_{A B}$ & $p_{B} \alpha_{A} C_{A B}+p_{A} \alpha_{B} C_{A B}-\frac{1}{2} \alpha_{A} \alpha_{B} C_{A B} C_{A_{2} B_{2}}$ \\
\hline OR & $p_{A}+p_{B}-p_{A} p_{B} C_{A B}$ & $\left(1-p_{B}\right) \lambda \alpha_{A}+\left(1-p_{A}\right) \lambda \alpha_{B}-\frac{1}{2} \lambda \alpha_{A} \alpha_{B} C_{A_{1} B_{1}}$ \\
$Y=A+B$ & & \\
\hline
\end{tabular}


$\left|\begin{array}{c|c}\mathrm{XOR} \\ Y=A \oplus B\end{array}\right| p_{A}+p_{B}-2 p_{A} p_{B} C_{A B}\left(\lambda_{B}+p_{B}\left(\lambda_{A}-\lambda_{B}\right)\right) \alpha_{A}+\left(\lambda_{A}+p_{A}\left(\lambda_{B}-\lambda_{A}\right)\right) \alpha_{B}-\left(\lambda_{A} C_{A_{1} B_{2}}+\lambda_{B} C_{A_{2} B_{1}}\right) \alpha_{A} \alpha_{B} \mid$

\subsection{Calculation of Correlation Factors}

The purpose of this subsection is to provide methods for calculating/propagating correlation factors through basic elements of a circuit. For two signals $A$ and $B$, there are two kinds of correlations that need to be established: probability correlation factor donated as $C_{A B}$ (corresponding to correlation factor between event $A$ and event $B$ ) and transition correlation donated as $C_{A_{i} B_{j}}$ (corresponding to correlation factor between event $A_{i}$ and event $B_{j}$ ), where $i, j \in\{1,2\}$ and $A_{i}$ and $B_{j}$ are transition events corresponding to signal $A$ and signal $B$ respectively as shown in Figure 3.

The first rule to be established is the fan-out rule associated with the circuit diagram in Figure 6.

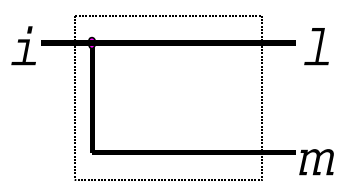

Figure 6. The circuit diagram associated with the fan-out rule.

Because signal $l$ is the same signal as $m$,

$$
\begin{gathered}
P(l)=P(m)=P(i) \\
\because P(l m)=P(l / m) P(m) \text { and } P(l / m)=1 \\
\therefore C_{l m}=\frac{P(l m)}{P(l) P(m)}=\frac{1}{P(l)}=\frac{1}{P(i)}
\end{gathered}
$$




$$
\begin{gathered}
P\left(l_{1}\right)=P\left(m_{1}\right)=P\left(i_{1}\right) \\
\because P\left(l_{1} / m_{1}\right)=1 \\
\therefore C_{l_{1} m_{1}}=\frac{P\left(l_{1} / m_{1}\right)}{P\left(l_{1}\right)}=\frac{1}{P\left(i_{1}\right)} \\
=\frac{2\left(1-p_{i}\right)}{\alpha_{i}}
\end{gathered}
$$

Similar,

$$
\begin{aligned}
& C_{l_{2} m_{2}}=\frac{1}{P\left(i_{2}\right)}=\frac{2 p_{i}}{\alpha_{i}} \\
& C_{l_{1} m_{2}}=0 \\
& C_{l_{2} m_{1}}=0
\end{aligned}
$$

The second rule is named AND rule and is associated with the circuit diagram in Figure 7.

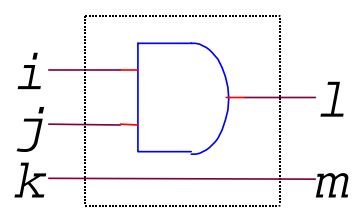

Figure 7. The circuit diagram associated with the AND rule.

Given correlation factors between input signals $i, j$ and $k$, the correlation factors between output signals $l$ and $m$ can be derived by follows:

Because $P(\operatorname{lm})=P(l / m) P(m)$ and using the results in Table 4, $P(l)=P(i) P(j) C_{i j}$,

$$
\begin{gathered}
P(l / m)=P(i / m) P(j / m) C_{i j} \\
=P(i / k) P(j / k) C_{i j} \\
=P(i) C_{i k} P(j) C_{j k} C_{i j} \\
=P(l) C_{i k} C_{j k} \\
\therefore C_{l m}=C_{i k} C_{j k} \\
P\left(l_{1} m_{1}\right)=P\left(l_{1}\right) P\left(m_{1}\right) C_{l_{1} m_{1}}=P\left(l_{1} / m_{1}\right) P\left(m_{1}\right)
\end{gathered}
$$


so

$$
\begin{aligned}
& C_{l_{1} m_{1}}=\frac{P\left(l_{1} / m_{1}\right)}{P\left(l_{1}\right)} \\
& P(\bar{l}) P\left(l_{1}\right)=P(00) P(00 \rightarrow 11)+P(01) P(01 \rightarrow 11)+P(10) P(10 \rightarrow 11) \\
& =P(\bar{i} \bar{j}) P\left(i_{1} j_{1}\right)+P(\bar{i} j) P\left(i_{1} \overline{j_{2}}\right)+P(\bar{i} \bar{j}) P\left(\overline{i_{2}} j_{1}\right) \\
& =\left(1-P(i)-P(j)+P(i) P(j) C_{i j}\right) P\left(i_{1}\right) P\left(j_{1}\right) C_{i_{1} j_{1}} \\
& +\left(P(j)-P(i) P(j) C_{i j}\right)\left(P\left(i_{1}\right)-P\left(i_{1}\right) P\left(j_{2}\right) C_{i_{1} j_{2}}\right) \\
& +\left(P(i)-P(i) P(j) C_{i j}\right)\left(P\left(j_{1}\right)-P\left(i_{2}\right) P\left(j_{1}\right) C_{i_{2} j_{1}}\right) \\
& P(\bar{l}) P\left(l_{1} / m_{1}\right)=\left(1-P(i)-P(j)+P(i) P(j) C_{i j}\right) P\left(i_{1} / m_{1}\right) P\left(j_{1} / m_{1}\right) C_{i_{1} j_{1}} \\
& +\left(P(j)-P(i) P(j) C_{i j}\right)\left(P\left(i_{1} / m_{1}\right)-P\left(i_{1} / m_{1}\right) P\left(j_{2} / m_{1}\right) C_{i_{1} j_{2}}\right) \\
& +\left(P(i)-P(i) P(j) C_{i j}\right)\left(P\left(j_{1} / m_{1}\right)-P\left(i_{2} / m_{1}\right) P\left(j_{1} / m_{1}\right) C_{i_{2} j_{1}}\right) \\
& =\left(1-P(i)-P(j)+P(i) P(j) C_{i j}\right) P\left(i_{1}\right) C_{i_{1} m_{1}} P\left(j_{1}\right) C_{j_{1} m_{1}} C_{i_{1} j_{1}} \\
& +\left(P(j)-P(i) P(j) C_{i j}\right)\left(P\left(i_{1}\right) C_{i_{1} m_{1}}-P\left(i_{1}\right) C_{i_{1} m_{1}} P\left(j_{2}\right) C_{j_{2} m_{1}} C_{i_{1} j_{2}}\right) \\
& +\left(P(i)-P(i) P(j) C_{i j}\right)\left(P\left(j_{1}\right) C_{j_{1} m_{1}}-P\left(i_{2}\right) C_{i_{2} m_{1}} P\left(j_{1}\right) C_{j_{1} m_{1}} C_{i_{2} j_{1}}\right) \\
& =\frac{1}{2} \lambda_{i} p_{j} \alpha_{i} C_{i_{1} m_{1}}+\frac{1}{2} \lambda_{j} p_{i} \alpha_{j} C_{j_{1} m_{1}} \\
& -\frac{1}{4}\left(\lambda_{i} C_{i_{1} m_{1}} C_{j_{2} m_{1}} C_{i_{1} j_{2}}+\lambda_{j} C_{i_{2} m_{1}} C_{j_{1} m_{1}} C_{i_{2} j_{1}}-\lambda C_{i_{1} m_{1}} C_{j_{1} m_{1}} C_{i_{1} j_{1}}\right) \alpha_{i} \alpha_{j}
\end{aligned}
$$

So

$$
C_{l_{1} m_{1}}=\frac{P\left(l_{1} / m_{1}\right)}{P\left(l_{1}\right)}=\frac{P(\bar{l}) P\left(l_{1} / m_{1}\right)}{P(\bar{l}) P\left(l_{1}\right)} .
$$

Solving Eg. (32) by applying Eqs. 30 and 31,

$$
\begin{aligned}
C_{l_{1} m_{1}}= & \frac{2}{\alpha(l)}\left(\begin{array}{l}
\frac{1}{2} \lambda_{i} p_{j} \alpha_{i} C_{i_{1} m_{1}}+\frac{1}{2} \lambda_{j} p_{i} \alpha_{j} C_{j_{1} m_{1}} \\
-\frac{1}{4}\left(\lambda_{i} C_{i_{1} m_{1}} C_{j_{2} m_{1}} C_{i_{1} j_{2}}+\lambda_{j} C_{i_{2} m_{1}} C_{j_{1} m_{1}} C_{i_{2} j_{1}}-\lambda C_{i_{1} m_{1}} C_{j_{1} m_{1}} C_{i_{1} j_{1}}\right) \alpha_{i} \alpha_{j}
\end{array}\right) \\
= & \lambda_{i} p_{j} C_{i_{1} k_{1}} \frac{\alpha_{i}}{\alpha_{l}}+\lambda_{j} p_{i} C_{j_{1} k_{1}} \frac{\alpha_{j}}{\alpha_{l}} \\
& -\frac{1}{2}\left(\lambda_{i} C_{i_{1} k_{1}} C_{j_{2} k_{1}} C_{i_{1} j_{2}}+\lambda_{j} C_{i_{2} k_{1}} C_{j_{1} k_{1}} C_{i_{2} j_{1}}-\lambda C_{i_{1} k_{1}} C_{j_{1} k_{1}} C_{i_{1} j_{1}}\right) \frac{\alpha_{i} \alpha_{j}}{\alpha_{l}}
\end{aligned}
$$


Other correlation factors (i.e., $C_{l_{1} m_{2}}, C_{l_{2} m_{1}}$, and $C_{l_{2} m_{2}}$ ) can be obtained similarly:

$$
\begin{aligned}
& C_{l_{1} m_{2}}=\frac{P\left(l_{1} / m_{2}\right)}{P\left(l_{1}\right)}=\frac{P(\bar{l}) P\left(l_{1} / m_{2}\right)}{P(\bar{l}) P\left(l_{1}\right)} \\
& =\lambda_{i} p_{j} C_{i_{1} k_{2}} \frac{\alpha_{i}}{\alpha_{l}}+\lambda_{j} p_{i} C_{j_{1} k_{2}} \frac{\alpha_{j}}{\alpha_{l}} \\
& -\frac{1}{2}\left(\lambda_{i} C_{i_{1} k_{2}} C_{j_{2} k_{2}} C_{i_{1} j_{2}}+\lambda_{j} C_{i_{2} k_{2}} C_{j_{1} k_{2}} C_{i_{2} j_{1}}-\lambda C_{i_{1} k_{2}} C_{j_{1} k_{2}} C_{i_{1} j_{1}}\right) \frac{\alpha_{i} \alpha_{j}}{\alpha_{l}} \\
& P\left(l_{2}\right)=P(11 \rightarrow 00)+P(11 \rightarrow 01)+P(11 \rightarrow 10) \\
& =1-P(11 \rightarrow 11) \\
& =P\left(i_{2}\right)+P\left(j_{2}\right)-P\left(i_{2}\right) P\left(j_{2}\right) C_{i_{2} j_{2}} \\
& P\left(l_{2} / m_{1}\right)=P\left(i_{2} / m_{1}\right)+P\left(j_{2} / m_{1}\right)-P\left(i_{2} / m_{1}\right) P\left(j_{2} / m_{1}\right) C_{i_{2} j_{2}} \\
& =P\left(i_{2}\right) C_{i_{2} m_{1}}+P\left(j_{2}\right) C_{j_{2} m_{1}}-P\left(i_{2}\right) C_{i_{2} m_{1}} P\left(j_{2}\right) C_{j_{2} m_{1}} C_{i_{2} j_{2}} \\
& C_{l_{2} m_{1}}=\frac{P\left(l_{2} / m_{1}\right)}{P\left(l_{2}\right)} \\
& =\frac{P\left(i_{2}\right) C_{i_{2} m_{1}}+P\left(j_{2}\right) C_{j_{2} m_{1}}-P\left(i_{2}\right) C_{i_{2} m_{1}} P\left(j_{2}\right) C_{j_{2} m_{1}} C_{i_{2} j_{2}}}{P\left(i_{2}\right)+P\left(j_{2}\right)-P\left(i_{2}\right) P\left(j_{2}\right) C_{i_{2} j_{2}}} \\
& =\frac{p_{B} \alpha_{A} C_{i_{2} k_{1}}+p_{A} \alpha_{B} C_{j_{2} k_{1}}-\frac{1}{2} \alpha_{A} \alpha_{B} C_{i_{2} k_{1}} C_{j_{2} k_{1}} C_{i_{2} j_{2}}}{p_{B} \alpha_{A}+p_{A} \alpha_{B}-\frac{1}{2} \alpha_{A} \alpha_{B} C_{i_{2} j_{2}}} \\
& C_{l_{2} m_{2}}=\frac{P\left(l_{2} / m_{2}\right)}{P\left(l_{2}\right)} \\
& =\frac{P\left(i_{2}\right) C_{i_{2} m_{2}}+P\left(j_{2}\right) C_{j_{2} m_{2}}-P\left(i_{2}\right) C_{i_{2} m_{2}} P\left(j_{2}\right) C_{j_{2} m_{2}} C_{i_{2} j_{2}}}{P\left(i_{2}\right)+P\left(j_{2}\right)-P\left(i_{2}\right) P\left(j_{2}\right) C_{i_{2} j_{2}}} \\
& =\frac{p_{B} \alpha_{A} C_{i_{2} k_{2}}+p_{A} \alpha_{B} C_{j_{2} k_{2}}-\frac{1}{2} \alpha_{A} \alpha_{B} C_{i_{2} k_{2}} C_{j_{2} k_{2}} C_{i_{2} j_{2}}}{p_{B} \alpha_{A}+p_{A} \alpha_{B}-\frac{1}{2} \alpha_{A} \alpha_{B} C_{i_{2} j_{2}}}
\end{aligned}
$$

Derivations of correlation factors for OR and XOR gates follow in a similar fashion. 


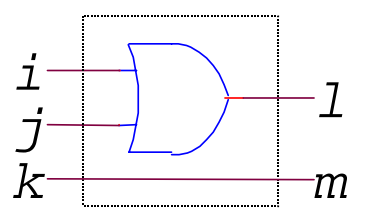

Figure 8. The circuit diagram associated with the OR rule.

Figure 8 shows the circuit diagram associated with the OR rule, and the correlation cofactors of the OR rule can be derived as follows:

$$
P\left(l_{1} m_{1}\right)=P\left(l_{1}\right) P\left(m_{1}\right) C_{l_{1} m_{1}}=P\left(l_{1} / m_{1}\right) P\left(m_{1}\right)
$$

so

$$
\begin{aligned}
& C_{l_{1} m_{1}}=\frac{P\left(l_{1} / m_{1}\right)}{P\left(l_{1}\right)} \\
& P\left(l_{1}\right)=P(00 \rightarrow 01)+P(00 \rightarrow 11)+P(00 \rightarrow 10) \\
& =1-P(00 \rightarrow 00) \\
& =P\left(i_{1}\right)+P\left(j_{1}\right)-P\left(i_{1}\right) P\left(j_{1}\right) C_{i_{1} j_{1}} \\
& P\left(l_{1} / m_{1}\right)=P\left(i_{1} / m_{1}\right)+P\left(j_{1} / m_{1}\right)-P\left(i_{1} / m_{1}\right) P\left(j_{1} / m_{1}\right) C_{i_{1} j_{1}} \\
& =P\left(i_{1}\right) C_{i_{1} m_{1}}+P\left(j_{1}\right) C_{j_{1} m_{1}}-P\left(i_{1}\right) C_{i_{1} m_{1}} P\left(j_{1}\right) C_{j_{1} m_{1}} C_{i_{1} j_{1}} \\
& =P\left(i_{1}\right) C_{i_{1} k_{1}}+P\left(j_{1}\right) C_{j_{1} k_{1}}-P\left(i_{1}\right) C_{i_{1} k_{1}} P\left(j_{1}\right) C_{j_{1} k_{1}} C_{i_{1} j_{1}} \\
& C_{l_{1} m_{1}}=\frac{P\left(l_{1} / m_{1}\right)}{P\left(l_{1}\right)} \\
& =\frac{P\left(i_{1}\right) C_{i_{1} k_{1}}+P\left(j_{1}\right) C_{j_{1} k_{1}}-P\left(i_{1}\right) C_{i, k_{1}} P\left(j_{1}\right) C_{j_{1} k_{1}} C_{i_{1} j_{1}}}{P\left(i_{1}\right)+P\left(j_{1}\right)-P\left(i_{1}\right) P\left(j_{1}\right) C_{i_{1} j_{1}}} \\
& =\frac{\left(1-p_{j}\right) \alpha_{i} C_{i_{1} k_{1}}+\left(1-p_{i}\right) \alpha_{j} C_{j_{1} k_{1}}-\frac{1}{2} \alpha_{i} \alpha_{j} C_{i_{i} k_{1}} C_{j, k_{1}} C_{i_{1} j_{1}}}{\left(1-p_{j}\right) \alpha_{i}+\left(1-p_{i}\right) \alpha_{j}-\frac{1}{2} \alpha_{i} \alpha_{j} C_{i_{1} j_{1}}} \\
& C_{l_{1} m_{2}}=\frac{P\left(l_{1} / m_{2}\right)}{P\left(l_{1}\right)} \\
& =\frac{\left(1-p_{j}\right) \alpha_{i} C_{i_{1} k_{2}}+\left(1-p_{i}\right) \alpha_{j} C_{j_{1} k_{2}}-\frac{1}{2} \alpha_{i} \alpha_{j} C_{i_{1} k_{2}} C_{j_{1} k_{2}} C_{i_{1} j_{1}}}{\left(1-p_{j}\right) \alpha_{i}+\left(1-p_{i}\right) \alpha_{j}-\frac{1}{2} \alpha_{i} \alpha_{j} C_{i_{1} j_{1}}}
\end{aligned}
$$




$$
\begin{aligned}
P(Y) P\left(Y_{2}\right)= & P(01) P(01 \rightarrow 00)+P(10) P(10 \rightarrow 00)+P(11) P(11 \rightarrow 00) \\
= & P(01) P\left(\overline{i_{1}} j_{2}\right)+P(10) P\left(i_{2} \overline{j_{1}}\right)+P(11) P\left(i_{2} j_{2}\right) \\
= & P(01) P\left(j_{2}\right)-P(01) P\left(i_{1}\right) P\left(j_{2}\right) C_{i_{1} j_{2}} \\
& +P(10) P\left(i_{2}\right)-P(10) P\left(i_{2}\right) P\left(j_{1}\right) C_{i_{2} j_{1}} \\
& +P(11) P\left(i_{2}\right) P\left(j_{2}\right) C_{i_{2} j_{2}} \\
= & \left(p_{j}-p_{i} p_{j} C_{i j}\right) \frac{\alpha_{j}}{2 p_{j}}-\left(p_{j}-p_{i} p_{j} C_{i j}\right)\left(\frac{\alpha_{i}}{2\left(1-p_{i}\right)} \frac{\alpha_{j}}{2 p_{j}}\right) C_{i_{i} j_{2}} \\
& +\left(p_{i}-p_{i} p_{j} C_{i j}\right) \frac{\alpha_{i}}{2 p_{i}}-\left(p_{i}-p_{i} p_{j} C_{i j}\right)\left(\frac{\alpha_{j}}{2\left(1-p_{j}\right)} \frac{\alpha_{i}}{2 p_{i}}\right) C_{i_{2} j_{1}} \\
& +p_{i} p_{j} C_{i j} \frac{\alpha_{i}}{2 p_{i}} \frac{\alpha_{j}}{2 p_{j}} C_{i_{2} j_{2}} \\
= & \frac{1}{2}\left(1-p_{j}\right) \lambda_{j} \alpha_{i}+\frac{1}{2}\left(1-p_{i}\right) \lambda_{i} \alpha_{j}-\frac{1}{4}\left(\lambda_{i} C_{i_{1} j_{2}}+\lambda_{j} C_{i_{2} j_{1}}-C_{i j} C_{i_{2} j_{2}}\right) \alpha_{i} \alpha_{j} \\
= & \frac{1}{2} \alpha_{l}
\end{aligned}
$$

$$
\begin{aligned}
P(l) P\left(l_{2} / m_{1}\right)= & P(01) P\left(j_{2} / m_{1}\right)-P(01) P\left(i_{1} / m_{1}\right) P\left(j_{2} / m_{1}\right) C_{i_{1} j_{2}} \\
& +P(10) P\left(i_{2} / m_{1}\right)-P(10) P\left(i_{2} / m_{1}\right) P\left(j_{1} / m_{1}\right) C_{i_{2} j_{1}} \\
& +P(11) P\left(i_{2} / m_{1}\right) P\left(j_{2} / m_{1}\right) C_{i_{2} j_{2}} \\
= & P(01) P\left(j_{2}\right) C_{j_{2} m_{1}}-P(01) P\left(i_{1}\right) C_{i_{i} m_{1}} P\left(j_{2}\right) C_{j_{2} m_{1}} C_{i_{1} j_{2}} \\
& +P(10) P\left(i_{2}\right) C_{i_{2} m_{1}}-P(10) P\left(i_{2}\right) C_{i_{2} m_{1}} P\left(j_{1}\right) C_{j_{1} m_{1}} C_{i_{2} j_{1}} \\
& +P(11) P\left(i_{2}\right) C_{i_{2} m_{1}} P\left(j_{2}\right) C_{j_{2} m_{1}} C_{i_{2} j_{2}} \\
= & \frac{1}{2}\left(1-p_{j}\right) \lambda_{j} \alpha_{i} C_{i_{2} m_{1}}+\frac{1}{2}\left(1-p_{i}\right) \lambda_{i} \alpha_{j} C_{j_{2} m_{1}} \\
& -\frac{1}{4}\left(\lambda_{i} C_{i_{1} j_{2}} C_{i_{1} m_{1}} C_{j_{2} m_{1}}+\lambda_{j} C_{i_{2} j_{1}} C_{i_{2} m_{1}} C_{j_{1} m_{1}}-C_{i j} C_{i_{2} j_{2}} C_{i_{2} m_{1}} C_{j_{2} m_{1}}\right) \alpha_{i} \alpha_{j}
\end{aligned}
$$




$$
\begin{aligned}
& \frac{1}{2}\left(1-p_{j}\right) \lambda_{j} \alpha_{i} C_{i_{2} m_{1}}+\frac{1}{2}\left(1-p_{i}\right) \lambda_{i} \alpha_{j} C_{j_{2} m_{1}} \\
C_{l_{2} m_{1}}= & \frac{-\frac{1}{4}\left(\lambda_{i} C_{i_{1} j_{2}} C_{i_{1} m_{1}} C_{j_{2} m_{1}}+\lambda_{j} C_{i_{2} j_{1}} C_{i_{2} m_{1}} C_{j_{1} m_{1}}-C_{i j} C_{i_{2} j_{2}} C_{i_{2} m_{1}} C_{j_{2} m_{1}}\right) \alpha_{i} \alpha_{j}}{\frac{1}{2} \alpha_{l}} \\
= & \left(1-p_{j}\right) \lambda_{j} C_{i_{2} m_{1}} \frac{\alpha_{i}}{\alpha_{l}}+\left(1-p_{i}\right) \lambda_{i} C_{j_{2} m_{1}} \frac{\alpha_{j}}{\alpha_{l}} \\
- & \frac{1}{2}\left(\lambda_{i} C_{i_{1} j_{2}} C_{i_{1} m_{1}} C_{j_{2} m_{1}}+\lambda_{j} C_{i_{2} j_{1}} C_{i_{2} m_{1}} C_{j_{1} m_{1}}-C_{i j} C_{i_{2} j_{2}} C_{i_{2} m_{1}} C_{j_{2} m_{1}}\right) \frac{\alpha_{i} \alpha_{j}}{\alpha_{l}} \\
C_{l_{2} m_{2}}= & \left(1-p_{j}\right) \lambda_{j} C_{i_{2} m_{2}} \frac{\alpha_{i}}{\alpha_{l}}+\left(1-p_{i}\right) \lambda_{i} C_{j_{2} m_{2}} \frac{\alpha_{j}}{\alpha_{l}} \\
- & \frac{1}{2}\left(\lambda_{i} C_{i_{1} j_{2}} C_{i_{1} m_{2}} C_{j_{2} m_{2}}+\lambda_{j} C_{i_{2} j_{1}} C_{i_{2} m_{2}} C_{j_{1} m_{2}}-C_{i j} C_{i_{2} j_{2}} C_{i_{2} m_{2}} C_{j_{2} m_{2}}\right) \frac{\alpha_{i} \alpha_{j}}{\alpha_{l}}
\end{aligned}
$$

Figure 9 shows the Circuit diagram associated with the XOR rule, followed by the derivation of the correlation factors.

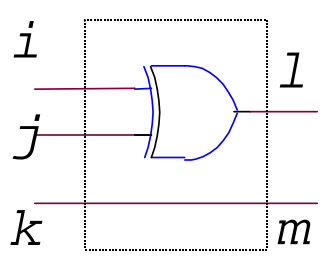

Figure 9. The circuit diagram associated with the XOR rule.

$$
\begin{gathered}
P(l)=P(i)+P(j)-2 P(i) P(j) C_{i j} \\
P(l / m)=P(i / m)+P(j / m)-2 P(i / m) P(j / m) C_{i j} \\
=P(i) C_{i m}+P(j) C_{j m}-2 P(i) C_{i m} P(j) C_{j m} C_{i j} \\
C_{l m}=\frac{P(l / m)}{P(l)} \\
=\frac{P(i) C_{i m}+P(j) C_{j m}-2 P(i) C_{i m} P(j) C_{j m} C_{i j}}{P(i)+P(j)-2 P(i) P(j) C_{i j}} \\
=\frac{P(i) C_{i k}+P(j) C_{j k}-2 P(i) C_{i k} P(j) C_{j k} C_{i j}}{P(i)+P(j)-2 P(i) P(j) C_{i j}}
\end{gathered}
$$




$$
\begin{aligned}
P(\bar{l}) P\left(l_{1}\right) & =P(00) P(00 \rightarrow 01)+P(00) P(00 \rightarrow 10)+P(11) P(11 \rightarrow 10)+P(11) P(11 \rightarrow 01) \\
& =P(00)\left(P\left(\overline{i_{1}} j_{1}\right)+P\left(i_{1} \overline{j_{1}}\right)+P(11)\left(P\left(\overline{i_{2}} j_{2}\right)+P\left(i_{2} \overline{j_{2}}\right)\right.\right. \\
& =\frac{1}{2}\left(\left(1-p_{j}\right) \lambda \alpha_{i}+\left(1-p_{i}\right) \lambda \alpha_{j}-\lambda \alpha_{i} \alpha_{j} C_{i_{1} j_{1}}\right)+\frac{1}{2}\left(p_{j} \alpha_{i}+p_{i} \alpha_{j}-\alpha_{i} \alpha_{j} C_{i_{2} j_{2}}\right) C_{i j} \\
& =\frac{1}{2}\left(\left(1-p_{j}\right) \lambda+p_{j} C_{i j}\right) \alpha_{i}+\frac{1}{2}\left(\left(1-p_{i}\right) \lambda+p_{i} C_{i j}\right) \alpha_{j}-\frac{1}{2}\left(\lambda C_{i_{1} j_{1}}+C_{i j} C_{i_{2} j_{2}}\right) \alpha_{i} \alpha_{j} \\
& =\frac{1}{2} \alpha_{l}
\end{aligned}
$$

$$
\begin{aligned}
& P(\bar{l}) P\left(l_{1} / m_{1}\right)=\frac{1}{2}\left(\left(1-p_{j}\right) \lambda C_{i_{1} m_{1}}+p_{j} C_{i j} C_{i_{2} m_{1}}\right) \alpha_{i}+\frac{1}{2}\left(\left(1-p_{i}\right) \lambda C_{j_{1} m_{1}}+p_{i} C_{i j} C_{j_{2} m_{1}}\right) \alpha_{j} \\
& -\frac{1}{2}\left(\lambda C_{i_{1} j_{1}} C_{i_{1} m_{1}} C_{j_{1} m_{1}}+C_{i j} C_{i_{2} j_{2}} C_{i_{2} m_{1}} C_{j_{2} m_{1}}\right) \alpha_{i} \alpha_{j} \\
& C_{l_{1} m_{1}}=\frac{P\left(l_{1} / m_{1}\right)}{P\left(l_{1}\right)} \\
& \frac{1}{2}\left(\left(1-p_{j}\right) \lambda C_{i_{1} m_{1}}+p_{j} C_{i j} C_{i_{2} m_{1}}\right) \alpha_{i}+\frac{1}{2}\left(\left(1-p_{i}\right) \lambda C_{j_{1} m_{1}}+p_{i} C_{i j} C_{j_{2} m_{1}}\right) \alpha_{j} \\
& =\frac{-\frac{1}{2}\left(\lambda C_{i_{1} j_{1}} C_{i_{1} m_{1}} C_{j_{1} m_{1}}+C_{i j} C_{i_{2} j_{2}} C_{i_{2} m_{1}} C_{j_{2} m_{1}}\right) \alpha_{i} \alpha_{j}}{\frac{1}{2} a_{l}} \\
& =\left(\left(1-p_{j}\right) \lambda C_{i_{1} m_{1}}+p_{j} C_{i j} C_{i_{2} m_{1}}\right) \frac{\alpha_{i}}{a_{l}}+\left(\left(1-p_{i}\right) \lambda C_{j_{1} m_{1}}+p_{i} C_{i j} C_{j_{2} m_{1}}\right) \frac{\alpha_{j}}{a_{l}} \\
& -\left(\lambda C_{i_{1} j_{1}} C_{i_{1} m_{1}} C_{j_{1} m_{1}}+C_{i j} C_{i_{2} j_{2}} C_{i_{2} m_{1}} C_{j_{2} m_{1}}\right) \frac{\alpha_{i} \alpha_{j}}{a_{l}} \\
& C_{l_{1} m_{2}}=\frac{P\left(l_{1} / m_{2}\right)}{P\left(l_{2}\right)} \\
& =\left(\left(1-p_{j}\right) \lambda C_{i_{1} m_{2}}+p_{j} C_{i j} C_{i_{2} m_{2}}\right) \frac{\alpha_{i}}{a_{l}}+\left(\left(1-p_{i}\right) \lambda C_{j_{1} m_{2}}+p_{i} C_{i j} C_{j_{2} m_{2}}\right) \frac{\alpha_{j}}{a_{l}} \\
& -\left(\lambda C_{i_{1} j_{1}} C_{i_{1} m_{2}} C_{j_{1} m_{2}}+C_{i j} C_{i_{2} j_{2}} C_{i_{2} m_{2}} C_{j_{2} m_{2}}\right) \frac{\alpha_{i} \alpha_{j}}{a_{l}} \\
& P(l) P\left(l_{2}\right)=P(01) P(01 \rightarrow 00)+P(01) P(01 \rightarrow 11)+P(10) P(10 \rightarrow 00)+P(10) P(10 \rightarrow 11) \\
& =P(01)\left(P\left(\overline{i_{1}} j_{2}\right)+P\left(i_{1} \overline{j_{2}}\right)\right)+P(10)\left(P\left(i_{2} \overline{j_{1}}\right)+P\left(\overline{i_{2}} j_{1}\right)\right) \\
& =\frac{1}{2} \alpha_{l}
\end{aligned}
$$




$$
\begin{aligned}
& P(l) P\left(l_{2} / m_{1}\right)=\left(\frac{1}{2} p_{j} \lambda_{i} C_{i_{1} m_{1}}+\frac{1}{2}\left(1-p_{j}\right) \lambda_{j} C_{i_{2} m_{1}}\right) \alpha_{i}+\left(\frac{1}{2} p_{i} \lambda_{j} C_{j_{l} m_{1}}+\frac{1}{2}\left(1-p_{i}\right) \lambda_{i} C_{j_{2} m_{1}}\right) \alpha_{j} \\
& -\frac{1}{2}\left(\lambda_{i} C_{i_{i} j_{2}} C_{i_{1} m_{1}} C_{j_{2} m_{1}}+\lambda_{j} C_{i_{2} j_{1}} C_{i_{2} m_{1}} C_{j_{1} m_{1}}\right) \alpha_{i} \alpha_{j} \\
& \left(\frac{1}{2} p_{j} \lambda_{i} C_{i_{1} m_{1}}+\frac{1}{2}\left(1-p_{j}\right) \lambda_{j} C_{i_{2} m_{1}}\right) \alpha_{i}+\left(\frac{1}{2} p_{i} \lambda_{j} C_{j_{1} m_{1}}+\frac{1}{2}\left(1-p_{i}\right) \lambda_{i} C_{j_{2} m_{1}}\right) \alpha_{j} \\
& C_{l_{2} m_{1}}=\frac{-\frac{1}{2}\left(\lambda_{i} C_{i_{1} j_{2}} C_{i_{1} m_{1}} C_{j_{2} m_{1}}+\lambda_{j} C_{i_{2} j_{1}} C_{i_{2} m_{1}} C_{j_{1} m_{1}}\right) \alpha_{i} \alpha_{j}}{\frac{1}{2} \alpha_{l}} \\
& =\left(p_{j} \lambda_{i} C_{i_{1} m_{1}}+\left(1-p_{j}\right) \lambda_{j} C_{i_{2} m_{1}}\right) \frac{\alpha_{i}}{\alpha_{l}}+\left(p_{i} \lambda_{j} C_{j m_{1}}+\left(1-p_{i}\right) \lambda_{i} C_{j_{2} m_{1}}\right) \frac{\alpha_{j}}{\alpha_{l}} \\
& -\left(\lambda_{i} C_{i_{1} j_{2}} C_{i_{1} m_{1}} C_{j_{2} m_{1}}+\lambda_{j} C_{i_{2} j_{1}} C_{i_{2} m_{1}} C_{j_{1} m_{1}}\right) \frac{\alpha_{i} \alpha_{j}}{\alpha_{l}} \\
& C_{l_{2} m_{1}}=\left(p_{j} \lambda_{i} C_{i_{1} m_{2}}+\left(1-p_{j}\right) \lambda_{j} C_{i_{2} m_{2}}\right) \frac{\alpha_{i}}{\alpha_{l}}+\left(p_{i} \lambda_{j} C_{j_{1} m_{2}}+\left(1-p_{i}\right) \lambda_{i} C_{j_{2} m_{2}}\right) \frac{\alpha_{j}}{\alpha_{l}} \\
& -\left(\lambda_{i} C_{i_{1} j_{2}} C_{i_{1} m_{2}} C_{j_{2} m_{2}}+\lambda_{j} C_{i_{2} j_{1}} C_{i_{2} m_{2}} C_{j_{1} m_{2}}\right) \frac{\alpha_{i} \alpha_{j}}{\alpha_{l}}
\end{aligned}
$$

Finally, Figure 10 is the Circuit diagram associated with the NOT rule, followed by the correlation factor derivation.

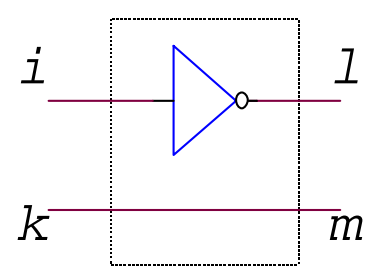

Figure 10. The circuit diagram associated with the NOT rule.

$$
\begin{aligned}
& P\left(l_{1}\right)=P\left(i_{2}\right) \\
& P\left(l_{1} / m_{1}\right)=P\left(i_{2} / m_{1}\right)=P\left(i_{2}\right) C_{i_{2} m_{1}} \\
& C_{l_{1} m_{1}}=C_{i_{2} m_{1}}=C_{i_{2} k_{1}} \\
& C_{l_{1} m_{2}}=C_{i_{2} m_{2}}=C_{i_{2} k_{2}}
\end{aligned}
$$




$$
\begin{aligned}
& P\left(l_{2}\right)=P\left(i_{1}\right) \\
& P\left(l_{2} / m_{1}\right)=P\left(i_{1} / m_{1}\right)=P\left(i_{1}\right) C_{i_{1} m_{1}} \\
& C_{l_{2} m_{1}}=C_{i_{1} k_{1}} \\
& C_{l_{2} m_{2}}=C_{i_{1} k_{2}} \\
& C_{l_{1} m_{2}}=C_{i_{2} k_{2}}
\end{aligned}
$$

The results of these basic rules used to propagate correlation factors from the inputs to the output

\begin{tabular}{|c|c|c|}
\hline Rules & Probability Correlation Factors & Transition Correlation Factors \\
\hline $\begin{array}{l}\text { Independent rule } \\
i \longleftarrow m \\
\\
\end{array}$ & $C_{l m}=C_{i j}$ & $C_{l_{1} m_{1}}=C_{i_{1} j_{1}}$ \\
\hline$i \stackrel{\text { Fan-out rule }}{\prod^{1}} 1$ & $C_{l m}=\frac{1}{P(i)}$ & $\begin{array}{l}C_{l_{1} m_{1}}=\frac{2\left(1-p_{i}\right)}{\alpha_{i}} \\
C_{l_{2} m_{2}}=\frac{2 p_{i}}{\alpha_{i}} \\
C_{l_{1} m_{2}}=0 \\
C_{l_{2} m_{1}}=0\end{array}$ \\
\hline \begin{tabular}{l|l|l}
$i$ & \\
$j$ & & \\
$k$ & &
\end{tabular} & $C_{l m}=C_{i k} C_{j k}$ & $\begin{array}{l}C_{l_{1} m_{1}}=\lambda_{i} p_{j} C_{i_{1} k_{1}} \frac{\alpha_{i}}{\alpha_{l}}+\lambda_{j} p_{i} C_{j_{1} k_{1}} \frac{\alpha_{j}}{\alpha_{l}} \\
-\frac{1}{2}\left(\begin{array}{l}\lambda_{i} C_{i_{1} k_{1}} C_{j_{2} k_{1}} C_{i_{1} j_{2}}+\lambda_{j} C_{i_{2} k_{1}} C_{j_{1} k_{1}} C_{i_{2} j_{1}} \\
-\lambda C_{i_{1} k_{1}} C_{j_{1} k_{1}} C_{i_{1} j_{1}}\end{array}\right) \frac{\alpha_{i} \alpha_{j}}{\alpha_{l}} \\
C_{l_{l} m_{2}}=\lambda_{i} p_{j} C_{i_{1} k_{2}} \frac{\alpha_{i}}{\alpha_{l}}+\lambda_{j} p_{i} C_{j_{1} k_{2}} \frac{\alpha_{j}}{\alpha_{l}} \\
-\frac{1}{2}\left(\begin{array}{l}\lambda_{i} C_{i_{1} k_{2}} C_{j_{2} k_{2}} C_{i_{1} j_{2}}+\lambda_{j} C_{i_{2} k_{2}} C_{j_{1} k_{2}} C_{i_{2} j_{1}} \\
-\lambda C_{i_{1} k_{2}} C_{j_{1} k_{2}} C_{i_{1} j_{1}}\end{array}\right) \frac{\alpha_{i} \alpha_{j}}{\alpha_{l}}\end{array}$ \\
\hline
\end{tabular}
are listed in Table 5. These basic rules along with the transformations for determining the Markov chain parameters for the output of a logic function (Table 3) are the foundational components for the algorithm developed in the next section.

Table 5. Set of basic rules used to calculate the output correlation factors. 


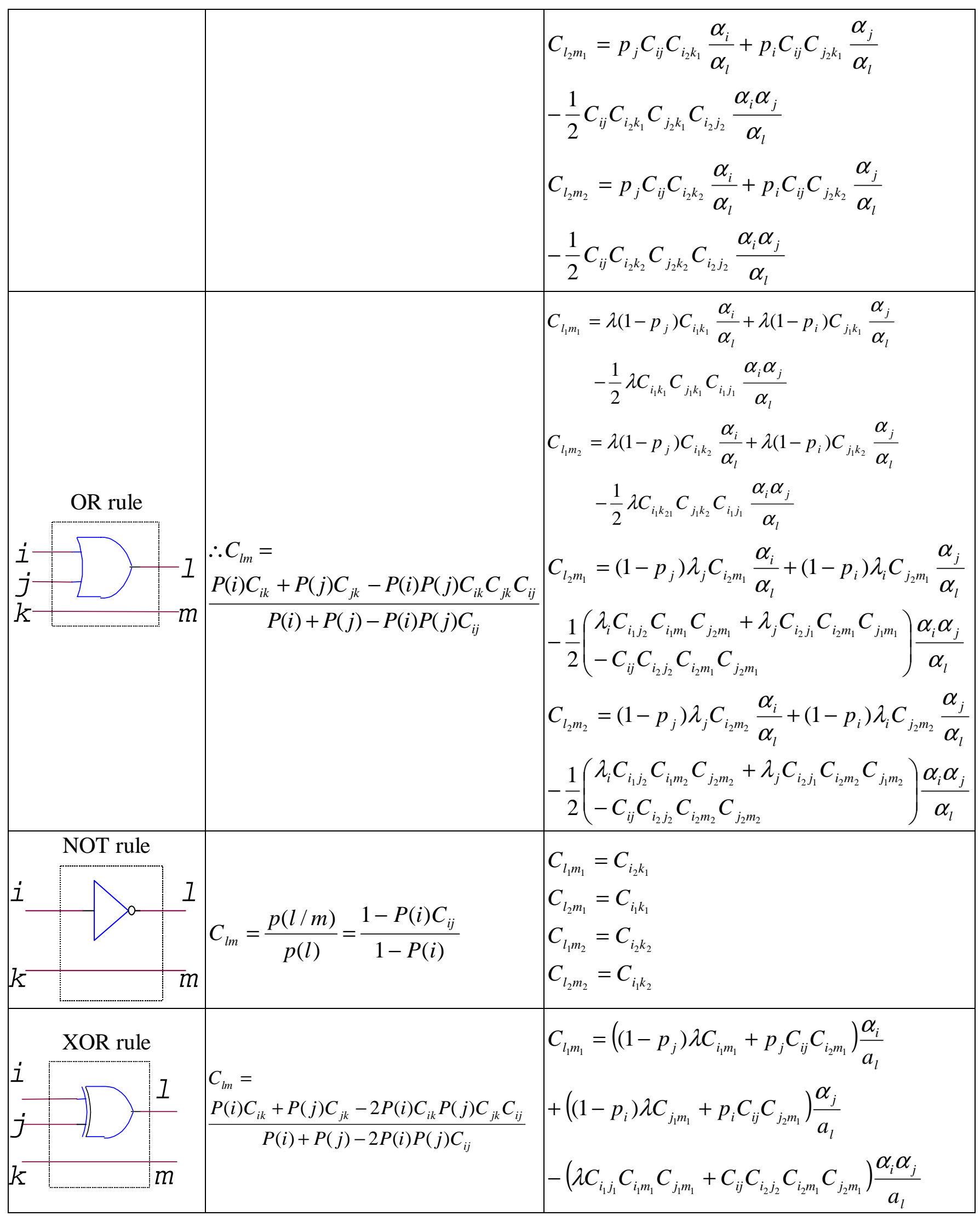




\begin{tabular}{|l|l}
$C_{l_{1} m_{2}}=\left(\left(1-p_{j}\right) \lambda C_{i_{1} m_{2}}+p_{j} C_{i j} C_{i_{2} m_{2}}\right) \frac{\alpha_{i}}{a_{l}}$ \\
$+\left(\left(1-p_{i}\right) \lambda C_{j_{1} m_{2}}+p_{i} C_{i j} C_{j_{2} m_{2}}\right) \frac{\alpha_{j}}{a_{l}}$ \\
$-\left(\lambda C_{i_{1} j_{1}} C_{i_{1} m_{2}} C_{j_{1} m_{2}}+C_{i j} C_{i_{2} j_{2}} C_{i_{2} m_{2}} C_{j_{2} m_{2}}\right) \frac{\alpha_{i} \alpha_{j}}{a_{l}}$ \\
$C_{l_{2} m_{1}}=\left(p_{j} \lambda_{i} C_{i_{1} m_{1}}+\left(1-p_{j}\right) \lambda_{j} C_{i_{2} m_{1}}\right) \frac{\alpha_{i}}{\alpha_{l}}$ \\
$+\left(p_{i} \lambda_{j} C_{j_{1} m_{1}}+\left(1-p_{i}\right) \lambda_{i} C_{j_{2} m_{1}}\right) \frac{\alpha_{j}}{\alpha_{l}}$ \\
$-\left(\lambda_{i} C_{i_{1} j_{2}} C_{i_{1} m_{1}} C_{j_{2} m_{1}}+\lambda_{j} C_{i_{2} j_{1}} C_{i_{2} m_{1}} C_{j_{1} m_{1}}\right) \frac{\alpha_{i} \alpha_{j}}{\alpha_{l}}$ \\
$C_{l_{2} m_{1}}=\left(p_{j} \lambda_{i} C_{i_{1} m_{2}}+\left(1-p_{j}\right) \lambda_{j} C_{i_{2} m_{2}}\right) \frac{\alpha_{i}}{\alpha_{l}}$ \\
$+\left(p_{i} \lambda_{j} C_{j_{1} m_{2}}+\left(1-p_{i}\right) \lambda_{i} C_{j_{2} m_{2}}\right) \frac{\alpha_{j}}{\alpha_{l}}$ \\
$-\left(\lambda C_{i_{1} j_{2}} C_{i_{1} m_{2}} C_{j_{2} m_{2}}+\lambda_{j} C_{i_{2} j_{1}} C_{i_{2} m_{2}} C_{j_{1} m_{2}}\right) \frac{\alpha_{i} \alpha}{\alpha_{l}}$
\end{tabular}

\section{Markov Chain Propagation Algorithm}

This section describes a proposed Markov Chain Propagation (MCP) algorithm for determining the Markov chain models for all signals of a given combinational circuit. The Markov chain signal model of Section 3 is employed, and it is assumed that the parameters of the model are known for the circuit's primary inputs. The overall approach is to propagate signal information associated with the Markov chain model through the circuit in a "gate-by-gate" fashion. Recall that once the Markov chain model is determined for all signals, the signal activities and circuit power estimate are determined using Eq. 13 and Eq. 1, respectively. It is assumed that the given circuit is specified at the level of basic logic gates. 


\section{MCP Algorithm}

Step 1: Represent the given combinational circuit as a directed acyclic graph (DAG).

Vertices of the DAG correspond to basic gates and edges represent signals. Two extra vertices

(a source and a sink) are included in the DAG to accommodate the primary inputs and outputs of the circuit. An example of how to represent a circuit with the DAG model is illustrated by Figures 11(a) and 11(b).

\section{Step 2: Perform a topological sort [10] on the DAG to obtain an ordering of the gates.}

See Figure 11(c).

Step 3: Transformation to two-input basic logic gates. As shown in Figure 11(d), replace all basic gates having more than two inputs with an equivalent sequence of two-input basic gates.

\section{Step 4 Partition the circuit into levels.}

As shown in Figure 11(e), levels are defined at the input and output of each basic gate. Note that there is at most one gate between any two consecutive levels.

\section{Step 5: $\quad$ Successively apply propagation rules at each level.}

Apply the propagation rules from Tables 3 and 5 for calculating the parameters of the Markov model for the basic gate outputs and the associated correlation factors.

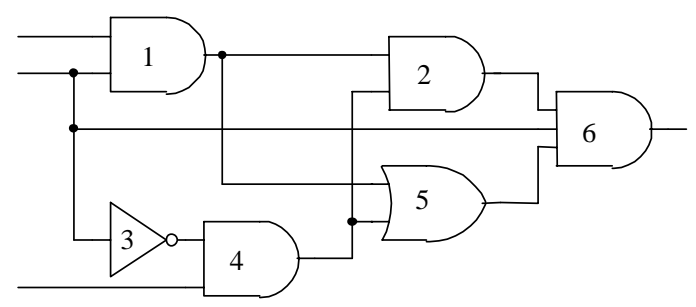

(a) 


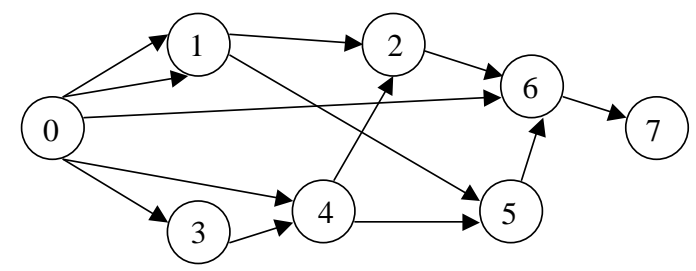

(b)

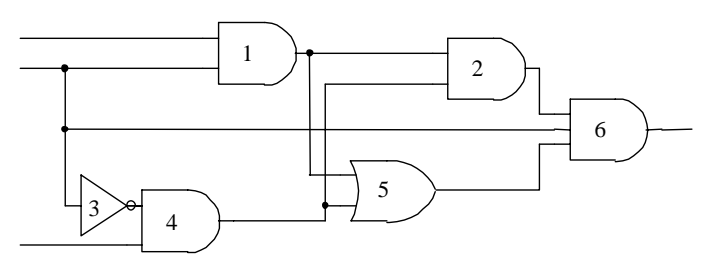

(c)

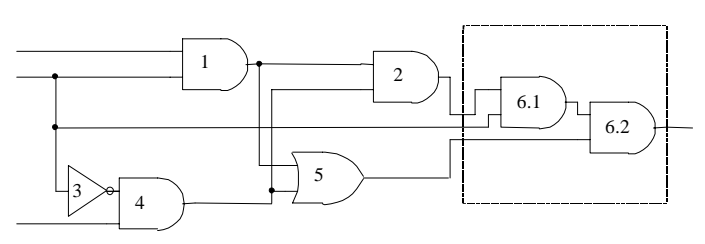

(d)

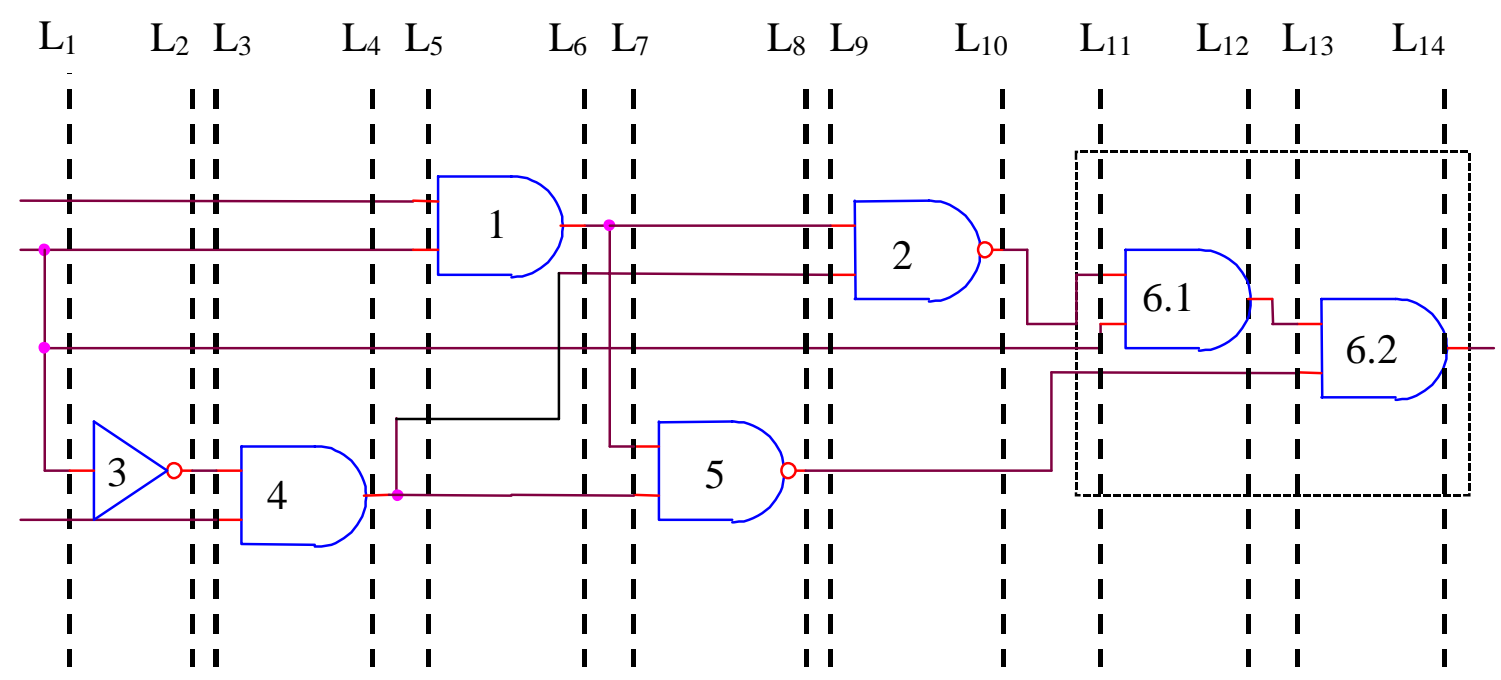

(e)

Figure 11. Illustration of the basic steps of the MCP Algorithm. 
In deriving the time complexity of the MCP algorithm, let $N$ denote the number of basic gates, $M$ be the number of fan-outs, and $S$ the number of physical signals. Fan-out is associated with a signal that is broadcast (i.e., duplicated). To illustrate, for the circuit of Figure 11(e), $N=7, M=7, S=17$. Because two levels are associated with each gate (one is placed before the gate and the other after), there are $2 N$ levels for a circuit with $N$ gates, which is 14 levels for the example shown in Figure 11(e).

Constructing the DAG (Step 1) from the given circuit requires $O(N+S)$ operations and it is shown in [10] that topological sort (Step 2) also requires $O(N+S)$ operations. Step 3 can be finished with no more than $S$ operations and at most $2 N$ operations are needed for Step 4.

For Step 5, there are two cases: from level $L_{i}$ to level $L_{i+1}$ and from level $L_{i+1}$ to level $L_{i+2}$, where $i=$ $1,3, \ldots, 2 N-1$. For the first case, because there is only one gate (e.g., gate 1 when $i=5$ as shown in Figure 11(e)) between level $L_{i}$ and level $L_{i+1}$, the calculation needed is to propagate the inputs of the single gate to the output of that gate. As shown in Figure 11(e), when $i=5$, the three parameters of the output signal of gate 1 can be obtained in a constant number of operations, denoted by $C_{1}$. The correlation factors between this output signal and other signals need to be calculated and inserted to the correlation factor table during this step. Because of the following three facts, it follows that the number of operations needed for this case of Step 5 can be expressed as $C_{1}+2 S C_{2}$ :

(i) only those signals having correlations with the input signals of the gate will have correlations with the output signal of the gate need to be calculated;

(ii) the maximum length of the correlation table of every entry is no more than $S$; and

(iii) the correlation factors between two signals can be done in a constant number of operations (assumed to be $C_{2}$ ) using basic rules shown in Table 5 . 
For the other case there isn't a gate between level $L_{i+1}$ to level $L_{i+2}$ (e.g., as shown in Figure 11(e), when $i=5$, this corresponds to $L_{6}$ to $L_{7}$ ). The only calculation needed in this case is to calculate the correlation factors due to recovergent fan-outs. Assume there are $k_{i}$ fan-outs from level $L_{i+1}$ to level $L_{i+2}$. The needed number of operations is bounded by $k_{i} C_{2}$.

So the total number of operations in Step 5 is thereby

$$
\sum_{j=1}^{N}\left(k_{2 j-1} C_{2}+C_{1}+2 S C_{2}\right)=M C_{2}+N C_{1}+2 N S C_{2}=O(N S)
$$

Combining the derived complexity results of Step 1 to Step 5, the time complexity of this MCP Algorithm is $O(N S)$.

\section{Experimental Results}

The MCP Algorithm has been implemented and evaluated using several test circuits. To verify the accuracy of the results produced by the MCP algorithm, PSpice ${ }^{\circledR}$ circuit simulations were performed on the same test circuits. In the simulation studies, time-series realizations from the assumed Markov chain model for each primary input were used to drive the circuit simulation. Estimates of signal probabilities were derived from the simulations by counting the fraction of time each signal took on a value of unity. Estimates of signal activities were derived from the simulations by counting signal transitions.

Figure 12 shows a six-gate circuit used for initial testing and evaluation. The comparison between probability and activity values produced by the MCP Algorithm and those produced through simulation are provided in Table 6. 


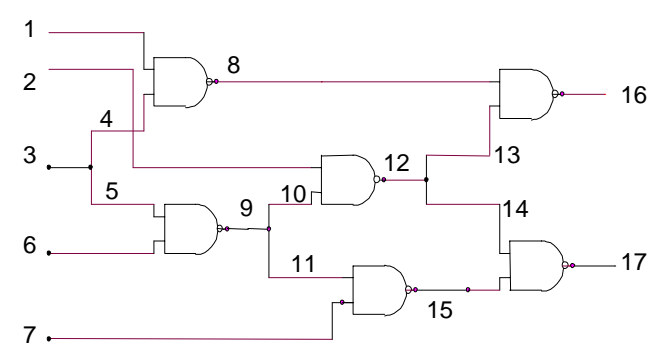

Figure 12. A simple test circuit.

Table 6. Results from MCP Algorithm and Simulation Studies for the circuit of Figure 12.

\begin{tabular}{|c|c|c|c|c|}
\hline \multirow{2}{*}{$\begin{array}{c}\text { Signal } \\
\text { No. }\end{array}$} & \multicolumn{2}{|c|}{ Signal Probability } & \multicolumn{2}{c|}{ Signal Activity } \\
\cline { 2 - 5 } & MCP & Simulation & MCP & Simulation \\
\hline 8 & 0.750 & 0.749 & 0.180 & 0.178 \\
\hline 9 & 0.750 & 0.747 & 0.180 & 0.182 \\
\hline 12 & 0.625 & 0.626 & 0.222 & 0.224 \\
\hline 15 & 0.625 & 0.630 & 0.222 & 0.223 \\
\hline 16 & 0.563 & 0.556 & 0.247 & 0.249 \\
\hline 17 & 0.563 & 0.560 & 0.254 & 0.255 \\
\hline
\end{tabular}

The MCP Algorithm was also evaluated using a circuit named C432 from the ISCAS-85 Benchmark Set. For this circuit there are a total of 145 distinct signals, not including the primary inputs. (Note that there are a total of 432 physical signals, which includes fan-out signals.) Table 7 show the distribution of absolute differences between activity values computed by the MCP Algorithm and those derived through simulation. These results indicate that the MCP Algorithm produces very accurate predictions of signal activities.

Table 7. Results from MCP Algorithm and Simulation Studies for Circuit C432 from the ISCAS-85 Benchmark Set.

\begin{tabular}{|c|c|}
\hline $\begin{array}{c}\text { Range of } \\
\text { Difference in } \\
\text { Activity Values }\end{array}$ & $\begin{array}{c}\text { Number of } \\
\text { Signals }\end{array}$ \\
\hline$[0,0.01]$ & 70 \\
\hline$(0.01,0.02]$ & 35 \\
\hline
\end{tabular}




\begin{tabular}{|c|c|}
\hline$(0.02,0.03]$ & 19 \\
\hline$(0.03,0.04]$ & 10 \\
\hline$(0.04,0.05]$ & 10 \\
\hline$(0.05,0.06]$ & 1 \\
\hline$(0.06,1]$ & 0 \\
\hline
\end{tabular}

\section{Summary and Future Work}

The problem of determining the activities of all signals of a combinational circuit is addressed in this paper. A new signal model is proposed based on a Markov chain. Signal activity is easily computed from the parameters associated with the proposed signal model. In the proposed approach, signals with known Markov chain representations are propagated through the circuit to produce a Markov chain representation for the output of each gate in the circuit. Accuracy of the approach is verified by comparing signal activities produced by the proposed method with corresponding activities produced through simulation studies. These initial testing results will be extended in future work by testing more and larger circuits.

The current circuit model assumes zero propagation delay through each gate. In reality, gates have non-zero delays, which results in "signal glitching." To illustrate how non-zero delays cause glitches, consider an example circuit as shown in Figure 13(a). Under the assumption of zero delay, the sample input signals $x_{1}, x_{2}$ and $x_{3}$ result in the output signals $y_{1}$ and $y_{2}$ as shown in Figure 13(b). Notice that output signal $y_{2}$ experiences no transitions. For non-zero delays (assume the delay of each gate is $d$ ) the output signal $y_{2}$ for the same inputs is derived and shown in Figure 13(c), which has several "glitching" transitions. Power consumption is impacted by these signal glitches; thus, future work is underway to extend the work presented in this report to consider the effect of glitches due to non-zero propagation delays. 


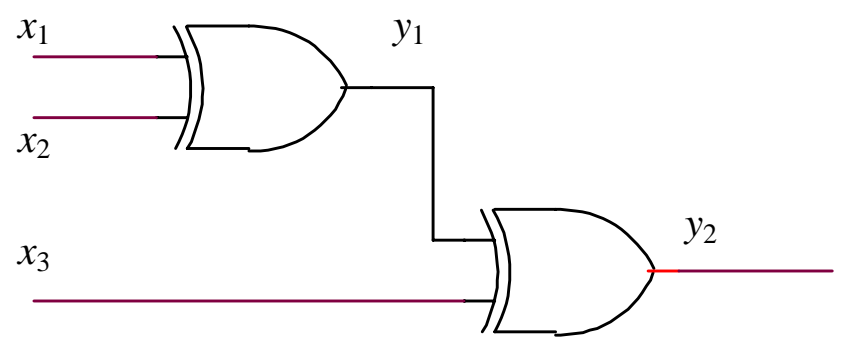

(a)

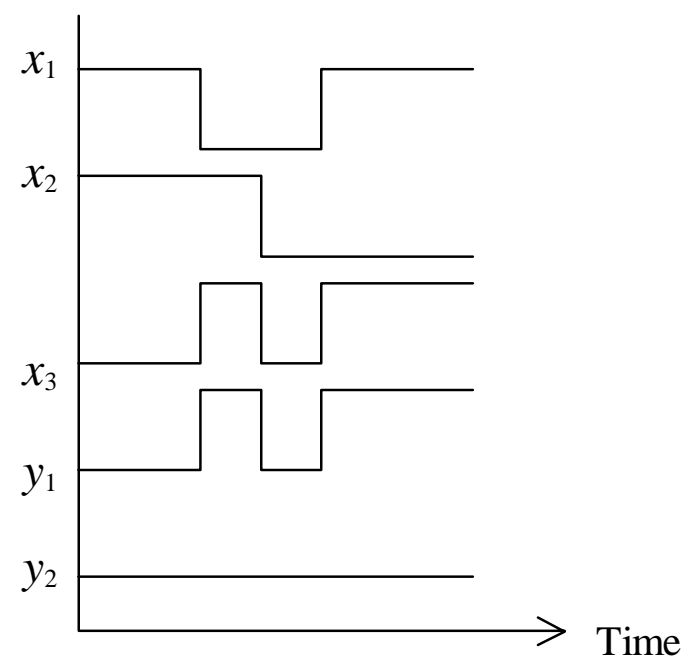

(b)

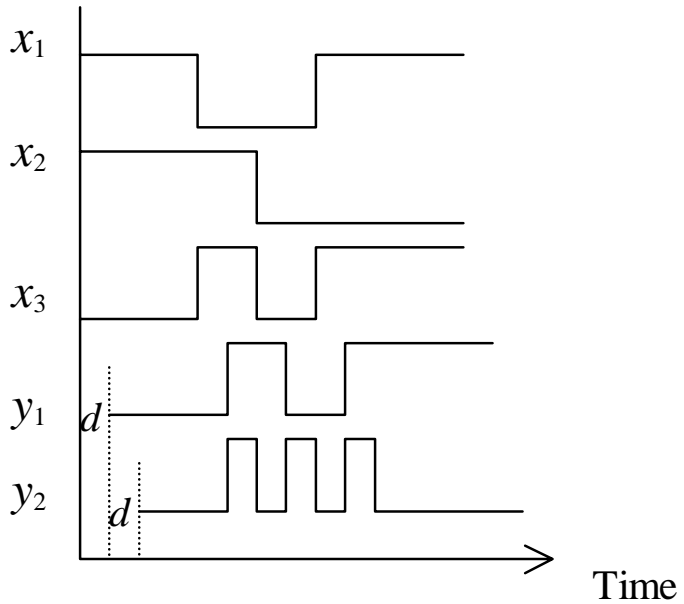

(c)

Figure 13. An example used to show how non-zero delays cause glitches. 


\section{Acknowledgments}

This research was supported by DARPA under Contract F30602-97-2-0297. The authors would like to thank Dr. S. Lakshmivarahan for his contributions to this work.

\section{References}

[1] R. Burch, F. N. Najm, P. Yang, and T. Trick, "A Monte Carlo Approach for Power Estimation", IEEE Trans. VLSI Systems, Vol. 1, No. 1, Mar. 1993, pp. 63-71.

[2] K. P. Parker and E. J. McCluskey, "Probabilistic Treatment of General Combinational Networks,” IEEE Trans. Computers, Vol. C-24, No. 6, June 1975, pp. 668-670.

[3] F. N. Najm, "Transition Density: A New Measure of Activity in Digital Circuits," IEEE Trans. Computer-Aided Design of Integrated Circuits and Systems, Vol. 12, No. 2, Feb. 1993, pp. 310323.

[4] T.-L. Chou and K. Roy, "Estimation of Activity for Static and Domino CMOS Circuits Considering Signal Correlations and Simultaneous Switching," IEEE Trans. Computer-Aided Design of Integrated Circuits and Systems, Vol. 15, No. 10, Oct. 1996, pp 1257-1265.

[5] F. N. Najm, "A Survey of Power Estimation Techniques in VLSI Circuits," IEEE Trans. on VLSI Systems, Vol. 2, No. 4, Dec. 1994, pp. 446-455.

[6] S. Ercolani, M. Favalli, M. Damiani, P. Olovo, and B. Ricco, "Estimate of Signal Probability in Combinational Logic Networks," Proc. IEEE European Test Conference, April 1989, pp. 132138. 
[7] B. Krishnamurthy and I. G. Tollis, "Improved Techniques for Estimating Signal Probabilities," IEEE Trans. Computers, Vol. 38, No. 7, July 1989, pp. 1041-1045.

[8] J. B. Thomas, An Introduction to Applied Probability and Random Processes, Krieger Publishing, Huntington, NY, 1981.

[9] M. J. M. Smith, Application-Specific Integrated Circuits, Addison Wesley, Reading, MA, 1997.

[10] T. H. Cormen, C. E. Leiserson, R. L. Rivest, and C. Stein, Introduction to Algorithms, McGrawHill New York, NY, 2001. 